\title{
Free boundary value problems for abstract elliptic equations and applications
}

\author{
Veli B. Shakhmurov \\ Department of Mechanical engineering, Okan University, Akfirat, Tuzla 34959 \\ Istanbul, Turkey, \\ E-mail: veli.sahmurov@okan.edu.tr
}

\begin{abstract}
Free bondary value problem for elliptic differential-operator equations with variable coefficients is studied. The uniform maximal regularity properties and Fredholmness of this problem are obtained in vector-valued Holder spaces.
\end{abstract}

\section{MSC 2010: 35xx, 47Fxx, 47Hxx, 35Pxx}

Key words: Free boundary value problems, Differential-operator equations, Banach-valued function spaces, Operator-valued multipliers, Interpolation of Banach spaces, Semigroup of operators.

\section{Introduction, notations and background}

In last years, the maximal regularity properties of boundary value problems (BVPs) for differential-operator equations (DOEs) have found many applications in PDE, psedo DE and in the different physical process (see for references [1 - 4], [6], [8], [10], [12-23], [27-28] ).

Let $\Omega$ be a domin in $R^{n}$ and $E$ is a Banach space. $C_{b}^{(m)}(\Omega ; E)$ will denote the spaces of $E$-valued bounded uniformly stongly continuous and $m$-times continuously differentiable functions on $\Omega$. For $m=0$ it denotes by $C_{b}(\Omega ; E)$. Let $\mathbb{C}$ denote the set of complex numbers. For $E=\mathbb{C}$ the space $C^{(m)}(\Omega ; E)$ will be denoted by $C_{b}^{(m)}(\Omega)$. Moreover, $C_{b}^{\infty}(\Omega ; E)$ denotes spaces of $E$-valued bounded strongly continiously differentiable functions of arbitrary order. We put $\mathbb{R}=(-\infty, \infty)$ and $\mathbb{R}_{+}=(0, \infty)$. Let $f(x)$ is a $E$-valued function and $f(x) \neq 0$. Consider

$$
\Omega_{f}=\left\{(x, y) \in \mathbb{R} \times \mathbb{R}_{+}, f \in C_{b}(\mathbb{R} ; E), 0<y<\|f(x)\|\right\} .
$$

The boundaries of $\Omega_{f}$ are given by

$$
\Gamma_{0}=\mathbb{R} \times 0, \Gamma_{f}=\left\{(x, y) \in \mathbb{R} \times \mathbb{R}_{+}, y=\|f(x)\|\right\} .
$$


Consider the following problem: Given $f_{0}, \nu \in C_{b}^{(2)}(\mathbb{R} ; E)$. Find a pair of functions $(u, f)$ possessing the regularity

$$
\begin{gathered}
f \in C_{b}^{(1)}\left(\left[0, T ; C_{b}^{(1)}(\mathbb{R} ; E)\right),\right. \\
u(t, .) \in W_{p}^{2}\left(\Omega_{f(t)} ; E\right), t \in J=[0, T) .
\end{gathered}
$$

and satisfying the following equations a.e.

$$
\begin{gathered}
-\Delta u(t, z)+A(x) u(t, z)=0, t \in J, z \in \Omega_{f(t)} \\
\frac{\partial u}{\partial y}=0, t \in J, z \in \Omega_{f(t)}, \\
u(t, z)=f(t, x), t \in J, z \in \Gamma_{f(t)}, \\
\lim _{z \rightarrow \infty} u(t, z)=\nu(t), t \in[0, T), \\
f_{t}(t, x)+\sqrt{1+f_{x}^{2}(t, x)} \frac{\partial}{\partial n} u(t, z)=0, t \in(0, T), z \in \Gamma_{f(t)}, \\
f(0, x)=f_{0}(x), x \in \mathbb{R},
\end{gathered}
$$

where $A$ is a linear operator in a Banach space $E$ and $z=(x, y)$ represents a generic point in $\bar{\Omega}_{f}$. Moreover, $\Delta$ denotes the Laplace operator with respect to the Euclidean metric, $\frac{\partial}{\partial n}$ denotes the derivative in direction of the outer unit normal $n$ at $\Gamma_{f(t)}$.

Maximal regularity properties of partial DOEs in $L_{p}$ spaces were studied in [1], [4], [7], [18-23]. The results in [4] and [18-23] were restricted to rectangular domain and equations that were not contained mixed derivatives in leading part. Moreover, problems investigated in [1] and [8] involve only bounded operator coefficients. In [18] the Dirichlet problem for the elliptic differential-operator equation of the second order in general domain was studied.

In contrast to all above we study general BVP (1.1) for equation with unbounded operator coefficients in the general domain.

Consider the BVP

$$
\begin{gathered}
L u=\sum_{i, j=1}^{n} a_{i j}(x) \frac{\partial^{2} u}{\partial x_{i} \partial x_{j}}-A(x) u(x)=F(x), \\
L_{1} u=\left.u\right|_{\Gamma}=0,
\end{gathered}
$$

where $\Gamma$ is a boundary of region $G \subset R^{n}$ and $a_{i j}$ are real-valued functions on $\bar{G}$.

We say that the problem (1.3) is maximal $H$-regular (or separable in Holder space $C^{\gamma}$ ) if:

(1) for all $F \in C^{\gamma}(G ; E)$ there exists a unique solution $u \in C^{2, \gamma}(G ; E(A), E)$ satisfying (1.3) a.e. on $G$; 
(2) there exists a positive constant $C$ independent of $F$ such that

$$
\sum_{i, j=1}^{n}\left\|\frac{\partial^{2} u}{\partial x_{i} \partial x_{j}}\right\|_{C^{\gamma}(G ; E)}+\|A u\|_{C^{\gamma}(G ; E)} \leq C\|F\|_{C^{\gamma}(G ; E)} .
$$

Let $G$ denote the operator generated by the problem (1.3) for $\lambda=0$, i.e.,

$$
\begin{gathered}
D(G)=C_{0}^{2, \gamma}(G ; E(A), E)=\left\{u \in C^{2, \gamma}(G ; E) \cap C(G ; E(A)),\right. \\
\left.\left.u\right|_{\Gamma}=0\right\}, G u=L u .
\end{gathered}
$$

The paper is organized as follows: Section1 collects definitions and background materials, embedding theorems of Sobolev-Lions spaces.

Let $\mathbb{C}$ be the set of complex numbers and

$$
S(\varphi)=\{\lambda \in \mathbb{C},|\arg \lambda| \leq \varphi\} \cup\{0\}, 0 \leq \varphi<\pi .
$$

Let $E_{1}$ and $E_{2}$ be two Banach spaces. $L\left(E_{1}, E_{2}\right)$ denotes the space of all bounded linear operators from $E_{1}$ to $E_{2}$. For $E_{1}=E_{2}=E$ it will be doneted by $L(E)$.

A linear operator $A$ is said to be $\varphi$-positive in a Banach space $E$ with bound $M>0$ if $D(A)$ is dense on $E$ and

$$
\left\|(A+\lambda I)^{-1}\right\|_{B(E)} \leq M(1+|\lambda|)^{-1}
$$

with $\lambda \in S(\varphi), \varphi \in[0, \pi)$, where $I$ is an identity operator in $E$.

Sometimes instead of $A+\lambda I$ will be written $A+\lambda$ and will denoted by $A_{\lambda}$. It is known that $([25, \S 1.15 .1])$ there exist fractional powers $A^{\theta}$ of positive operator $A$. Let $E\left(A^{\theta}\right)$ denote the space $D\left(A^{\theta}\right)$ with graphical norm

$$
\|u\|_{E\left(A^{\theta}\right)}=\left(\|u\|^{p}+\left\|A^{\theta} u\right\|^{p}\right)^{\frac{1}{p}}, 1 \leq p<\infty,-\infty<\theta<\infty .
$$

A linear operator $A(x)$ is said to be positive in $E$ uniformly in $x$ if $D(A(x))$ is independent of $x, D(A(x))$ is dense in $E$ and

$$
\left\|(A(x)+\lambda I)^{-1}\right\| \leq M(1+|\lambda|)^{-1}
$$

for all $\lambda \in S(\varphi)$ and $\varphi \in[0, \pi)$.

Let $\Omega$ be a domain in $R^{n}$. $C(\Omega, E)$ and $C^{m}(\Omega ; E)$ will denote the spaces of $E$-valued bounded uniformly strongly continuous and $m$-times continuously differentiable functions on $\Omega$, respectively.

Let $0<\gamma \leq 1$. $C^{\gamma}(\Omega ; E)$ denotes the space of $E$-valued strongly bounded continiuous functions that are defined on $\Omega \subset R^{n}$ with the norm

$$
\|f\|_{C^{\gamma}(\Omega ; E)}=\|f\|_{C^{\gamma}(\Omega ; E)}+[f]^{\gamma}(E),
$$


where

$$
[f]^{\gamma}(E)=\sup _{x \neq y, x, y \in \Omega} \frac{\|f(x)-f(y)\|_{E}}{|x-y|^{\gamma}} .
$$

$C^{\gamma, m}(\Omega ; E)$ denotes the space of $E$-valued strongly bounded continiuous functions that are defined on $\Omega \subset R^{n}$ with the norm

$$
\begin{gathered}
C^{\gamma, m}(\Omega ; E)=\left\{f \in C^{m}(\Omega ; E), f^{(m)} \in C^{\gamma}(\Omega ; E)\right\}, \\
\|f\|_{C^{\gamma, m}(\Omega ; E)}=\|f\|_{C^{m}(\Omega ; E)}+\left\|f^{(m)}\right\|_{C^{\gamma}(\Omega ; E)}<\infty .
\end{gathered}
$$

Let $E_{0}$ and $E$ be two Banach spaces and $E_{0}$ is continuously and densely embedded into $E$. Let $m$ be a natural number.

Let $\alpha=\left(\alpha_{1}, \alpha_{2}, \ldots, \alpha_{n}\right)$ are $n$ tuples of nonnegative integer numbers and

$$
D^{\alpha}=\frac{\partial^{|\alpha|}}{\partial x_{1}^{\alpha_{1}} \partial x_{2}^{\alpha_{2}} \ldots \partial x_{n}^{\alpha_{n}}} .
$$

$C^{m, \gamma}\left(\Omega ; E_{0}, E\right)$ denote the space of $E_{0}$-valued bounded uniformly stongly continuous and $m$-times continuously differentiable functions on $\Omega$ with norm

$$
\|f\|_{C^{m, \gamma}\left(\Omega ; E_{0}, E\right)}=\|f\|_{C^{m, \gamma}(\Omega ; E)}+\|f\|_{C^{\gamma}\left(\Omega ; E_{0}\right)} .
$$

For $E_{0}=E$ the space $C^{m, \gamma}\left(\Omega ; E_{0}, E\right)$ will denoted by $C^{m, \gamma}(\Omega ; E)$.

Let $A$ be a linear operator in a Banach space $E$ so that is a generator of analytic semigroup $U(t)=U_{A}(t)$. Let

$$
\begin{gathered}
D_{A}(\theta, p)=\left\{u \in E, \quad\|u\|_{D_{A}(\theta, p)}=\left\|t^{1-\theta-\frac{1}{p}} A U(t) u\right\|_{L^{p}(0,1 ; E)}<\infty\right\} \\
\text { for } 1 \leq p<\infty \text { and } \theta \in(0,1) ; \\
D_{A}(\theta, \infty)=\left\{u \in E, \quad\|u\|_{D_{A}(\theta, p)}=\left\|t^{1-\theta} A U(t) u\right\|_{L^{\infty}(0,1 ; E)}<\infty\right\} \\
\text { for } p=\infty \text { and } 0<\theta \leq 1 ; \\
D_{A}(\theta)=\left\{u \in D_{A}(\theta, \infty), \lim _{t \rightarrow 0} t^{1-\theta} A U(t) u=0\right\} .
\end{gathered}
$$

From [15, Proposition 2.2.2] we obtain the following

$$
\begin{gathered}
D_{A}(\theta, p)=(E, D(A))_{\theta, p}, \text { for } 1 \leq p<\infty, \theta \in(0,1), \\
D_{A}(\theta, \infty)=(E, D(A))_{\theta, \infty} \text { for } 0<\theta \leq 1, \\
D_{A}(\theta)=(E, D(A))_{\theta}, \text { for } \theta \in(0,1) .
\end{gathered}
$$

$H\left(E_{0}, E\right)$ denotes the class of linear operators that are isomorphisim from $E_{0}$ onto $E$ and are negative generators of stronge continious and analytic semigroups. 
Let $S^{\prime}\left(R^{n} ; E\right)$ denote the space of all continuous linear operators $L: S\left(R^{n} ; E\right) \rightarrow$ $E$, equipped with the bounded convergence topology. Recall $S\left(R^{n} ; E\right)$ is norm dense in $L^{p}\left(R^{n} ; E\right)$ when $1 \leq p<\infty$.

Let $L_{q}^{*}(E)$ denote the space of all $E$-valued functions $u(t)$ such that

$$
\|u\|_{L_{q}^{*}(E)}=\left(\int_{0}^{\infty}\|u(t)\|_{E}^{q} \frac{d t}{t}\right)^{\frac{1}{q}}<\infty, 1 \leq q<\infty,\|u\|_{L_{\infty}^{*}(E)}=\sup _{0<t<\infty}\|u(t)\|_{E} .
$$

Let $F$ denote the Fourier transform. Fourier-analytic definition of $E$-valued Besov space on $R^{n}$ are defined as in [25 $\S 3$ ], i.e.,

$$
\begin{gathered}
B_{p, q}^{s}\left(R^{n} ; E\right)=\left\{u \in S^{\prime}\left(R^{n} ; E\right),\right. \\
\|u\|_{B_{p, q}^{s}\left(R^{n} ; E\right)}=\left\|F^{-1} \sum_{k=1}^{n} t^{\varkappa_{k}-s_{k}}\left(1+\left|\xi_{k}\right|^{\varkappa_{k}}\right) e^{-t|\xi|^{2}} F u\right\|_{L_{q}^{*}\left(L_{p}\left(R^{n} ; E\right)\right)}, \\
\left.p, q \in[1, \infty], \varkappa_{k}>s_{k}, s=\left(s_{1}, s_{2}, \ldots, s_{n}\right)\right\} .
\end{gathered}
$$

For appropriate domain $\Omega \subset R^{n}$ the space $B_{p, q}^{s}(\Omega ; E)$ is defined as usulal restriction of the space $B_{p, q}^{s}\left(R^{n} ; E\right)$.

For $E=\mathbb{C}$ the space $B_{p, q}^{s}(\Omega ; \mathbb{C})$ will be denoted by $B_{p, q}^{s}(\Omega)$.

Let $h^{s}=h^{s}\left(R^{n} ; E\right)$ denote the closure of $S\left(R^{n} ; E\right)$ in $B_{\infty, \infty}^{s}\left(R^{n} ; E\right)$. Assume that $\Omega$ is an open subset of $R^{n}$ and let $r_{\Omega}$ denote the restriction operator with respect to $\Omega$, i.e., $r_{\Omega} u=\left.u\right|_{\Omega}$ for $B_{\infty, \infty}^{s}\left(R^{n} ; E\right)$. Here, $h^{s}(\Omega ; E)$ is defined as the closure of $r_{\Omega}\left(S\left(R^{n} ; E\right)\right)$ in $B_{\infty, \infty}^{\gamma}(\Omega ; E)$ and $h^{m, \gamma}(\Omega ; E)$ is defined as the closure of $r_{\Omega}(S(\Omega ; E))$ in $C^{m, \gamma}(\Omega ; E)$. For $E=\mathbb{C}$ the spaces $h^{s}(\Omega ; E), h^{m, \gamma}(\Omega ; E)$ will be denoted by $h^{s}$ and $h^{m, \gamma}$, respectively. Moreover, let $C_{0}^{s}(\Omega ; E)$ denote the closure of $C^{\infty}(\Omega ; E)$ in $C^{s}(\Omega ; E)$.

Let

$$
\begin{gathered}
h_{+}^{s}=h_{+}^{s}(\mathbb{R} ; E)=\left\{g \in h^{s}, g(x) \neq 0\right\}, \\
X=h^{\alpha}(Q ; E), X_{k}=h^{k, \alpha}(Q ; E), Y=h^{2, \alpha}(Q ; E(A), E)= \\
\left\{u \in h^{\alpha}(Q ; D(A)) \cap h^{2, \alpha}(Q ; E)\right\}
\end{gathered}
$$

with the norm

$$
\|u\|_{Y}=\|u\|_{h^{\alpha}(Q ; D(A))}+\left\|u^{(2)}\right\|_{h^{\alpha}(Q ; E)}<\infty .
$$

Here,

$$
B_{+}^{2+\alpha}=\left\{u \in B_{\infty, \infty}^{2+\alpha}\left(R^{n} ; E\right), g(x) \neq 0\right\}
$$

and

$$
h^{m, \alpha}(A)=h^{m, \alpha}\left(\mathbb{R} ; D_{A}(\alpha, \infty)\right), \alpha \in(0,1) .
$$


Remark 1.1. In order to formulate our result, let

$$
h_{\nu}^{s}=h_{\nu}^{s}(E)=\left\{\nu+g ; g \in h^{s}(\mathbb{R} ; E)\right\}, h_{\nu}^{s, \alpha}=\left\{\nu+g ; g \in h^{s, \alpha}(\mathbb{R} ; E)\right\}
$$

and $f \in h_{\nu}^{2, \alpha}$ given. Let $u_{f}$ denote the unique solution of the BVP

$$
-\Delta u+A u=0, \partial_{y} u=0 \text { on } \Gamma_{0}, u=f \text { on } \Gamma_{f},
$$

where $A$ is a linear operator in a Banach space $E$.

$$
k_{f}=\frac{\|f\|^{2}}{\left(1+\|f\|+\left\|f_{x}\right\|^{2}\right)\left(1+\left\|f_{x}\right\|^{2}\right)}
$$

and define

$$
V_{\nu}=\left\{f \in C_{b}^{2}(\mathbb{R} ; E)\right\}, f(x) \neq 0, \partial_{y} u_{f}(x, f(x))<k_{f} \text { for } x \in \mathbb{R} .
$$

It is clear to see that $u_{\nu} \equiv \nu$. Hence, $\nu \in V_{\nu}$. More precisely, by following [9, Lemma 5.10] it can be shown that $V_{\nu}$ is a open neighborhood of $\nu$ in $h_{\nu}^{s, 2}(E)$ and that

$$
\operatorname{diam}_{h^{2, s}}\left(V_{\nu}\right)=\sup _{g, v \in V_{\nu}}\|g-v\|_{h^{s, 2}}=\infty .
$$

Suppose now that $(u, f)$ is a classical solution of $(1.1)-(1.2)$. We call $(u, f)$ a classical Hölder solution on $J$ if it possesses the additional regularity

$$
f \in C\left(J ; V_{\nu}\right) \cap C^{1}\left(J ; h_{\nu}^{1, \alpha}\right), u(t, .) \in h_{\nu}^{2, \alpha}(\mathbb{R} ; E), t \in J .
$$

We will prove the following main result

Theorem 1. Given $f_{0} \in V_{\nu}$, there exist $t^{+}=t^{+}\left(f_{0}\right)$ and a unique maximal classical Hölder solution $(u, f)$ of problem $(1.1)-(1.2)$ on $\left[0, t^{+}\right)$. Moreover, the mapping $\left(t, f_{0}\right) \rightarrow f$ defines a local $C^{\infty}$-semiflow on $V_{\nu}$. If $t^{+}<\infty$ and $f$ : $\left[0, t^{+}\right) \rightarrow V_{\nu}$ is uniformly continuous then either

$$
\lim _{t \rightarrow t^{+}}\|f(t, .)\|_{h^{2, \alpha}}=\infty, \lim _{t \rightarrow t^{+}} \inf _{v \in V_{\nu}}\|f(t, .)-v\|_{h^{2, \alpha}}=0 .
$$

In the first stage, we transform problem (1.1) -(1.2) into a nonlinear problem on a fixed domain

$$
\frac{d f}{d t}+O(f)=0, f(0)=f_{0}
$$

with respect to only the unknown function $f$, which determines the free boundary $\Gamma_{f}$, where $O$ is a nonlinear operator in $E$.

Then, by using the solution of the above problem we will show the exsistence of regular solution of the free BVP (1.1) - (1.2).

\section{Transformed problem}


Let $\nu=\nu(t)>0$ be fixed. Define

$$
G_{\nu}=\left\{g \in C_{b}^{(2)}(\mathbb{R} ; E), \nu(t) I+g(x) \neq 0\right\},
$$

where $I$ is an identity element in the Banach space $E$.

Consider the following transformation

$$
(x, y)=\varphi\left(x^{\prime}, y^{\prime}\right)=\varphi_{g}\left(x^{\prime}, y^{\prime}\right)=\left(x^{\prime}, 1-\frac{y^{\prime}}{\nu+g\left(x^{\prime}\right)}\right), \text { for }\left(x^{\prime}, y^{\prime}\right) \in \Omega_{f} .
$$

It is easily verified that $\varphi_{g}$ is a diffeomorphism of class $C^{2}$ which maps $\Omega_{g}$ onto the strip $Q=\mathbb{R} \times(0,1)$. Moreover,

$$
\begin{gathered}
\left(x^{\prime}, y^{\prime}\right)=\varphi^{-1}(x, y)=\varphi_{g}^{-1}(x, y)=(x,(1-y) g(x)) \text { for }(x, y) \in Q . \\
\varphi_{*} u=\varphi_{*}^{g} u=u\left(\varphi_{g}^{-1}(x, y)\right) \text { for } u \in W_{p}^{2}\left(\Omega_{g} ; E(A), E\right) .
\end{gathered}
$$

Let $u$ be an $E$-valued function defined on $Q$. Here, $u_{\left.\right|_{\Gamma_{i}}}$ denote the restriction of $u$ on $\Gamma_{i}$, where

$$
\Gamma_{i}=\mathbb{R} \times\{i\}, \gamma_{i} u=u_{\mid \Gamma}, i=0,1 .
$$

Lemma 2.1. Given $g \in \Phi_{\eta}$ and $v \in C^{2, \gamma}(Q ; E(A), E)$; under the the maps (2.2) the operators in (1.1) are transformed into the following:

$$
\begin{gathered}
B(g) v=-\varphi^{g} *(\Delta+A)\left(\varphi_{g} * v\right) \text { on }[0, T) \times Q, \\
B_{i}(g) v=\varphi_{*}^{g}\left(\left(\nabla\left(\varphi_{g}^{*}\right), n_{i}\right)\right) \text { on }(0, T) \times \Gamma_{i},
\end{gathered}
$$

where $n_{0}=\left(-g_{x}, 1\right), n_{1}=(0,-1)$ denote the outer normals according to $\Gamma_{f}$ and $\Gamma_{0}$, i.e.,

$$
\begin{gathered}
B_{0}(g) v=\varphi_{*}^{g}\left(-\frac{\partial}{\partial x} \varphi_{g}^{*} v+\frac{\partial}{\partial y} \varphi_{g}^{*} v\right) \text { on }(0, T) \times \Gamma_{0}, \\
B_{1}(f) v=\varphi_{*}^{g}\left(-\frac{\partial}{\partial y} \varphi_{g}^{*} v\right) \text { on }[0, T) \times \Gamma_{1} .
\end{gathered}
$$

Lemma 2.2. Given $g \in \Phi_{\nu}$ and $v \in C^{2, \gamma}(Q ; E(A), E)$. Under the map (2.2) the problem (1.1) is transformed into the following:

$$
\begin{gathered}
B(f) v=-\varphi^{f} *(\Delta+A)\left(\varphi_{g} * v\right)=0 \text { on }[0, T) \times Q, \\
v=f \text { on }[0, T) \times \Gamma_{0}, \\
B_{1}(f) v=0 \text { on }[0, T) \times \Gamma_{1}, \\
\lim _{z \rightarrow \infty} v(t, z)=0, \text { on }[0, T), \\
\frac{\partial g}{\partial t}+B_{0}(g) v=0 \text { on }(0, T) \times \Gamma_{0},
\end{gathered}
$$




$$
g(0, .)=g_{0}(x) \text { on } \mathbb{R},
$$

A pair $(v, g)$ is called a solution of the problem (2.5) if

$$
\begin{gathered}
g \in C_{b}([0, T) ; \Phi) \cap C_{b}^{(1)}\left([0, T) ; C_{b}^{(1)}(\mathbb{R})\right), \\
u(t, .) \in W_{p}^{2}\left(\Omega_{f(t)} ; E\right), t \in[0, T)
\end{gathered}
$$

and $(v, g)$ satisfies $(2.5)$ a.e. on $[0, T) \times Q$.

Condition 2.1. Assume the following conditions are satisfied: $\sum_{i, j=1}^{2} a_{i j} \xi_{i} \xi_{j} \geq C|\xi|^{2}$, for $\xi=\left(\xi_{1}, \xi_{2}, \ldots, \xi_{n}\right) \in R^{n}$ and $C>0$

(2) operator $A$ is a positive operator in a Banach space $E$ for some $\varphi \in$ $(0, \pi]$.

In a similar way as in $[9$, Lemma 2.2$]$ we obtain

Lemma 2.3. Assume the Condition 2.1 are satisfied. Then for given $g \in \Phi$, we have

$$
\begin{gathered}
B(g) u=\sum_{i, j=1}^{2}-a_{i j}(g) \frac{\partial^{2} u}{\partial x_{i} \partial x_{j}}+a_{2}(g) \frac{\partial u}{\partial x_{2}}+A_{g} u, \\
B_{i}(g) u=\sum_{j=1}^{2} b_{j i}(g) \gamma_{i} \frac{\partial u}{\partial x_{j}}, i=0,1,
\end{gathered}
$$

and

$$
\sum_{i, j=1}^{2} a_{i j}(g) \xi_{i} \xi_{j} \geq \alpha(g)|\xi|^{2}, \text { for } \xi=\left(\xi_{1}, \xi_{2}, \ldots, \xi_{n}\right) \in R^{n},
$$

where $\alpha(g)>0, \gamma_{i}$ are trace operators from $Q$ to $\Gamma_{i}, i=0,1$,

$$
\begin{gathered}
a_{11}(g)=1, a_{12}(g)=a_{21}(g)=\frac{\beta g_{x_{1}}}{\nu+g}, a_{22}(g)=\frac{1+\beta^{2} g_{x_{1}}^{2}}{(\nu+g)^{2}}, \beta=1-x_{1}, \\
a_{2}(g)=\frac{\beta}{\nu+g}\left[\frac{2 g_{x_{1}}^{2}}{\nu+g}-g_{x_{1} x_{1}}\right], b_{10}(g)=-g_{x_{1}}, b_{20}(g)=-\frac{1+g_{x_{1}}^{2}}{\nu+g}, \quad(2.7) \\
b_{11}(g)=0, b_{21}(g)=\frac{1}{\nu+g}, \alpha(g)=\frac{1}{1+(\nu+g)^{2}+\beta^{2} g_{x_{1}}^{2}}, A(g)=A\left(\varphi_{g}\right) .
\end{gathered}
$$

\section{Abstract elliptic equation in the fixed domain}

In this section we study the elliptic BVP

$$
B(g) u=-\sum_{i, j=1}^{2} a_{i j}(g) \frac{\partial^{2} u}{\partial x_{i} \partial x_{j}}+a_{2}(g) \frac{\partial u}{\partial x_{2}}+A(x) u=f
$$




$$
B_{i}(g) u=\sum_{j=1}^{2} b_{j i}(g) \gamma_{i} \frac{\partial u}{\partial x_{j}}=f_{i}, i=0,1,
$$

where $B(g)$ and $B_{i}(g)$ are differential operators defined by $(2.6)$.

We will derive a priori estimates as well as isomorphism properties in framwork of abstract Holder spaces.

Condition 3.1. Assume the following conditions are satisfied:

(1) $a_{i j} \in C^{0, \alpha}(\bar{G}), a_{i j}=a_{j i}$;

(2) $\sum_{i, j=1}^{2} a_{i j}(g) \xi_{i} \xi_{j} \geq C(g)|\xi|^{2}$, for $\xi=\left(\xi_{1}, \xi_{2}, \ldots, \xi_{n}\right) \in R^{n}$ and $C(g)>0$;

(3) operator $A(g)$ is uniformly positive in a Banach algebra $E$ for some $\varphi \in(0, \pi]$.

Here $\partial B(g)[\psi, . v]$ denotes the Gateaux derivative of operator function $B(g)$ at $\psi$ in the direction of $v$.

Lemma 3.1. Suppose the Condition 3.1 is satisfied and $A(g)$ is Gateaux differentiable for $g \in h_{\Phi}^{2, \alpha}, \alpha \in(0,1)$. Then the map $g \rightarrow O_{0}(g)=\left\{B(g), B_{0}(g)\right\}$ is bounded linear operator-function from $Y$ into $X \times h^{1, \alpha}(A)$ and have continious derivatives of all order with respect to $g \in h_{\Phi}^{2, \alpha}$, i.e.

$$
B(.) \in C^{\infty}\left(h_{\Phi}^{2, \alpha} ; L(Y, X)\right), B_{0}(.) \in C^{\infty}\left(h_{\Phi}^{2, \alpha} ; L(Y, E)\right)
$$

and

$$
\begin{gathered}
\partial B(g)[u, v]=(\partial B(g) u) v=\frac{2 \beta}{\nu+g}\left\{\left(\frac{g_{x} u}{\nu+g}-u_{x}\right) v_{x_{1} x_{2}}+\frac{u}{(\nu+g)^{2}}\left(\left(\frac{1}{\beta}+\beta g_{x}^{2}\right)-\right.\right. \\
\left.\left.\frac{\beta}{\nu+g} g_{x} u_{x}\right) v_{x_{2} x_{2}}-\left(\frac{g_{x} u}{(\nu+g)^{2}}-\frac{g_{x x} u+4 g_{x} u_{x}}{2(\nu+g)}+\frac{u_{x x}}{2}\right) v_{x_{2}}\right\}+\partial A(g)[u, v], \\
\partial B_{0}(g)[u, v]=-u_{x} v_{x_{1}}+\frac{\beta}{\nu+g}\left[\frac{u\left(1+g_{x}^{2}\right)}{\nu+g}-2 g_{x} u_{x}\right] v_{x_{2}}
\end{gathered}
$$

for $g \in h_{+}^{2, \alpha}$ and $u, v \in Y$.

Proof. It is clear to see that $(\varphi, v) \rightarrow \varphi v$ is bilinear and continuous from $h^{s}$ into $Y$. Moreover, the mapping

$$
g \rightarrow \frac{1}{\nu+g} u
$$

are continious and are infinitely many differentible from $h^{s}$ into $Y$. By using the definition of the space $Y$ and Lemma 2.3 we get that for all fixed $g \in h_{\Phi}^{2, \alpha}$ the operator $u \rightarrow B(g)$ is bounded linear operator from $Y$ into $X$. So, we obtain that

$$
B(.) \in C^{\infty}\left(h_{\Phi}^{2, \alpha}, L(Y, X)\right) .
$$


Hence, in view of Lemma 2.3 we obtain

$$
B_{0}(.) \in C^{\infty}\left(h_{\Phi}^{2, \alpha}, L(Y, E)\right) \text {. }
$$

By using [4, Theorem 2] we obtain the following:

Theorem 3.1. Suppose the Condition 3.1 is satisfied and $\alpha \in(0,1)$. Then for $\lambda \in S(\varphi)$ and for sufficiently large $|\lambda|$ :

(a) the operator $u \rightarrow \tilde{O}(g) u=\left\{(B(g)+\lambda) u, \gamma_{0} u, \quad(\eta+g) B_{1}(g)\right\}$ is isomorphism from $Y$ onto $X \times h^{2, \alpha}(A) \times h^{1, \alpha}(A)$;

(b) for $\mu>0$ the operator $u \rightarrow\left\{(B(g)+\lambda) u, \mu \gamma_{0} u,(\eta+g) B_{1}(g)\right\}$ is isomorphism from $Y$ onto $X \times h^{2, \alpha}(A) \times h^{1, \alpha}(A)$;

(c) For $u \in Y$ there exists a positive constant $C$, depending only on $g, \eta, p$ and $E$ such that the coercive estimate holds:

$$
\|u\|_{Y} \leq C\left(\|(B(g)+\lambda) u\|_{X}+\left\|\gamma_{0} u\right\|_{h^{2, \alpha}(E)}+\left\|(\nu+g) B_{1} u\right\|_{h^{1, \alpha}(E)}\right) .
$$

Proof. Indeed, since the domain $Q$ is a strip, the functios $g, \eta$ are fixed smooth functions, by virtue of trace theorem in Hoder space $[15, \S 2]$ we obtain the assertion.

Consider now, the following BVPs

$$
\begin{gathered}
-\sum_{i, j=1}^{2} a_{i j}(g) \frac{\partial^{2} u}{\partial x_{i} \partial x_{j}}+a_{2}(g) \frac{\partial u}{\partial x_{2}}+A(g) u=F, \\
B_{i} u=\sum_{j=1}^{2} b_{j i}(g) \gamma_{i} \frac{\partial u}{\partial x_{j}}=0, i=0,1, \\
-\sum_{i, j=1}^{2} a_{i j}(g) \frac{\partial^{2} u}{\partial x_{i} \partial x_{j}}+a_{2}(g) \frac{\partial u}{\partial x_{2}}+A(g) u=0, \\
\sum_{j=1}^{2} b_{j 0}(g) \gamma_{0} \frac{\partial u}{\partial x_{j}}=\psi, \sum_{j=1}^{2} b_{j 1}(g) \gamma_{1} \frac{\partial u}{\partial x_{j}}=0 .
\end{gathered}
$$

Consider the operators $\tilde{S}(g)$ and $\tilde{K}(g)$ generated by problems $(3.4)-(3.5)$ and (3.6) - (3.7), respectively, i.e.

$$
\begin{gathered}
D(\tilde{S}(g))=\left\{u \in Y, B_{i} u=0, i=0,1\right\} \\
\tilde{S}(g) u=-\sum_{i, j=1}^{2} a_{i j}(g) \frac{\partial^{2} u}{\partial x_{i} \partial x_{j}}+a_{2}(g) \frac{\partial u}{\partial x_{2}}+A(g) u
\end{gathered}
$$

and

$$
D(\tilde{K}(g))=\left\{u \in Y, B u=0, B_{1} u=0\right\}, \tilde{K}(g) u=B_{0} u \in B_{0 p} .
$$


From the Theorem 3.5 we obtain that the inverse operators $\tilde{O}^{-1}(g), \tilde{S}^{-1}(g)$, $\tilde{K}^{-1}(g)$ are bounded from $X \times h^{2, \alpha}(A) \times h^{1, \alpha}(A), X, h^{2, \alpha}(E)$ into $Y$, respectively.

Here,

$$
O(g)=\tilde{O}^{-1}(g), S(g)=\tilde{S}^{-1}(g), K(g)=\tilde{K}^{-1}(g) .
$$

Assume $g \in h_{+}^{2, \alpha}(\mathbb{R} ; E), \psi \in D_{A}(\alpha)$, and put $u=K(g) v$. Then $u$ is the solution of the BVP (3.6) - (3.7) .

Condition 3.1. Assume $A(g)$ is Gateaux differentiable for $g \in h_{+}^{2, \alpha}, \alpha \in$ $(0,1)$ and the operator $\partial A(g)$ is uniformly $R$-positive in UMD (see e.g [8] for definitions) Banach algebra $E$.

Lemma 3.2. Suppose the conditions 3.1 and 3.2 are satisfied. Then we have

$$
K(.) \in C^{\infty}\left(h_{+}^{2+\alpha} ; L(E, Y)\right)
$$

and

$$
\partial K(g)[v, \psi]=-S(g) \partial B(g)[v, K(g) \psi]
$$

for $g \in h_{+}^{2, \alpha}, \psi \in E$ and $v \in Y$.

Proof. It follows from Lemma 3.1 and Theorem 3.1 that the map $g \rightarrow$ $\tilde{O}(g)$ is isomorphism from $Y$ onto $X \times h^{2, \alpha}(A) \times h^{1, \alpha}(A)$ and have continious derivatives of all order with respect to $g \in h_{+}^{2, \alpha}$, i.e.

$$
\tilde{O} \in C^{\infty}\left(h_{+}^{2, \alpha} ; \operatorname{Isom} Y, X \times h^{2, \alpha}(A) \times h^{1, \alpha}(A)\right)
$$

and

$$
\partial \tilde{O}(g) \psi=\{\partial B(g)[\psi, .], 0,0\} \text { for } \psi \in h^{2, \alpha}(A) .
$$

Then by reasoning as the Lemma 3.4 in [6] we obtain the assertion.

\section{The nonlinear operator for free BVPs}

In this section we introduce the basic nonlinear operator and we derive some properties of it.

Moreover, we show that the corresponding evolution problem involving this operator is equivalent to the original problem (1.2). Given $g \in h_{+}^{2, \alpha}$, we define the following operator

$$
O(g)=B_{0}(g) K(g) g .
$$

From lemmas 3.1 and 3.2 we get that

$$
O \in C^{\infty}\left(h_{+}^{2, \alpha}, h^{1, \alpha}(E)\right) .
$$

Assume that $g_{0} \in h_{+}^{2, \alpha}$, and let $\sigma=[0, T)$. A function $\sigma \rightarrow B_{10}$ is a classical solution of

$$
\frac{d g}{d t}+O(g)=0, g(0)=g_{0}
$$


iff $g \in C\left(\sigma ; h_{+}^{2, \alpha}\right) \cap C^{1}\left(\sigma ; h^{2, \alpha}(E)\right)$ and $g$ satisfies (4.2) pointwise.

Lemma 4.1. Suppose the Condition 3.1 is satisfied. Then for $g_{0} \in h_{+}^{2, \alpha}$ :

(a) Suppose that $g$ is a classical solution of problem (4.2) on $\sigma$ and let $v(t,)=.K(g(t,)) g.(t,$.$) . Then the pair (v, g)$ is a classical solution of $(2.5)$ on $\sigma$, having the additional regularity

$$
\begin{gathered}
g \in C\left(\sigma ; h_{U}^{2, \alpha}\right) \cap C^{1}\left(\sigma ; h^{1, \alpha}\right), \\
v(t, .) \in Y, t \in \sigma
\end{gathered}
$$

(b) Suppose that $(v, g)$ is a classical solution of (2.5) on $\sigma$, having the regularity (4.3). Then $g$ is a classical solution of (4.2) on $\sigma$.

Proof. The proof is obtained from Lemma 2.2 and definitions of the spaces $B_{i p}, i=0,1$ and $Y$.

For fixed $g \in h_{\Phi}$ consider the operator

$$
v \rightarrow B_{0}(g) K(g) v .
$$

In view of Theorem 3.1 we obtain that

$$
B_{0}(g) K(g) \in L\left(h^{2, \alpha}(A), h^{1, \alpha}(A)\right) .
$$

Lemma 4.2. Suppose the conditions 3.1 and 3.2 are satisfied. Then $O \in$ $C^{\infty}\left(h_{+}^{2, \alpha}, h^{1, \alpha}(A)\right)$ and

$$
\begin{gathered}
\partial O(g) \psi=B_{0}(g) K(g) \psi+\partial B_{0}(g)[v, K(g) \psi]- \\
B_{0}(g) S(g) \partial B(g)[v, K(g) \psi]
\end{gathered}
$$

for $g \in h_{+}^{2, \alpha}, \psi \in h^{2, \alpha}(A)$ and $v \in Y$.

Proof. The assertion is obtained from Lemma 3.1 and Lemma 3.2.

\section{Linear equation with constant coefficients}

We put

$$
R_{+}^{2}=\left\{x=\left(x_{1}, x_{2}\right) \in R^{2}, x_{2}>0\right\} .
$$

Consider the problem

$$
\begin{gathered}
-\sum_{i, j=1}^{2} a_{i j} \frac{\partial^{2} u}{\partial x_{i} \partial x_{j}}+A u+\mu^{2} u=0, \\
u\left(x_{1}, 0\right)=\psi\left(x_{1}\right), x \in R_{+}^{2},
\end{gathered}
$$


where $A=A(g)\left(x_{0}, 0\right), a_{i j}=a_{i j}(g)\left(x_{0}, 0\right)$ and $a_{i j}(g)$ are defined by $(2.7)$ and $\psi \in h^{2, \alpha}(A)$. By applying the Fourier transform to the problem $(5.1)-(5.2)$ with respect to $x_{1}$ we get

$$
\begin{gathered}
-a_{22} \frac{d^{2} \hat{u}}{d y^{2}}+2 a_{12} i \eta \frac{d \hat{u}}{d y}+\left(A+\eta^{2}+\mu^{2}\right) \hat{u}=0, \\
\hat{u}(\eta, 0)=\hat{\psi}(\eta), \eta \in \mathbb{R}, y \in \mathbb{R}_{+},
\end{gathered}
$$

where $x_{2}$ and $\hat{u}\left(\eta, x_{2}\right)$ are denoted by $y$ and $\hat{u}=\hat{u}(\eta, y)$, respectively.

Let

$$
p(\xi)=\xi_{1}^{2}+2 a_{12} \xi_{1} \xi_{2}+a_{22} \xi_{2}^{2} \text { for } \xi=\left(\xi_{1}, \xi_{2}\right) \in R^{2} .
$$

There exists an $\alpha>0$ with

$$
p(\xi) \geq \alpha|\xi|^{2} \text { for all } \xi \in R^{2} .
$$

The condition (5.5) implies that

$$
a_{12}^{2}-a_{22} \geq \alpha .
$$

Moreover, we define

$$
q_{\eta}(\mu, \lambda)=-a_{22} \lambda^{2}+2 a_{12} i \eta \lambda+\mu^{2}+\eta^{2}+1=0 .
$$

Remark 5.1. Given $\eta \in \mathbb{R}$ and $z \in \mathbb{C}$, then in view of (5.5) there is exactly one root of the equation (5.7) with positive real part. It is given by

$$
\lambda(\eta, \mu)=i a(\eta)+b(\eta, \mu),
$$

where

$$
a(\eta)=-\frac{a_{12}}{a_{22}} \eta, b(\eta, \mu)=\frac{1}{a_{22}}\left[a_{22}\left(1+\mu^{2}\right)+\left(a_{12}^{2}-a_{22}\right) \eta^{2}\right]^{\frac{1}{2}} .
$$

We put

$$
\begin{gathered}
\tilde{X}=h^{\alpha}\left(R_{+}^{2} ; E\right), \tilde{Y}=h^{2, \alpha}\left(R_{+}^{2} ; E(A) ; E\right), \\
N_{\mu}(\eta, y)=\exp \left\{-\lambda(\eta, \mu) A_{\mu}^{\frac{1}{2}}(\eta) y\right\} .
\end{gathered}
$$

The main result of this section is the following:

Theorem 5.1. Assume the Condition 3.1 is satisfied. Then problem (5.1)(5.2) has a unique solution $u \in \tilde{Y}$ for $\psi \in h^{2, \alpha}(A)$ and $u$ is represented by

$$
u\left(x_{1}, x_{2}\right)=\left(\tilde{K}_{0}+\mu^{2}\right) \psi=F^{-1} N_{\mu}\left(\eta, x_{2}\right) F \psi(\eta) .
$$

Moreover, the estimate holds

$$
\sum_{j=0}^{2}|\mu|^{2-j}\left\|\frac{\partial^{j} u}{\partial x_{1}^{j}}\right\|_{\tilde{X}}+\sum_{i, j=1}^{2}\left\|\frac{\partial^{2} u}{\partial x_{i} \partial x_{j}}\right\|_{\tilde{X}}+\|A u\|_{\tilde{X}} \leq C\|\psi\|_{h^{2, \alpha}(A)} .
$$


For the proof we need some preparation. Here,

$$
\begin{gathered}
A_{\mu}=A_{\mu}(\eta)=A+\mu^{2}+\eta^{2}, q_{0}(\eta, y)=A N_{\mu}(\eta, y), \\
q_{j}(\eta, y)=|\mu|^{2-j} \eta^{j} N_{\mu}(\eta, y) .
\end{gathered}
$$

We need the following lemmas:

Lemma 5.1. Assume the Condition 3.1 is satisfied. Then there exists a unique solution $\hat{u}(\eta, y)$ of $(5.3)-(5.4)$ expressing as

$$
\hat{u}(\eta, y)=N_{\mu}(\eta, y) \hat{\psi}(\eta)
$$

Moreover, the following uniformly in $\eta$ estimate holds

$$
\|A \hat{u}\|_{h^{\alpha}\left(\mathbb{R}_{+} ; E\right)}+\sum_{j=0}^{2}|\mu|^{2-j}\left\|\hat{u}^{(j)}\right\|_{h^{\alpha}\left(\mathbb{R}_{+} ; E\right)} \leq C\|\hat{\psi}\|_{h^{2, \alpha}(A)} .
$$

Proof. In view of positivity of operator $A$ we know that $-A_{\mu}^{\frac{1}{2}}(\eta)$ is analytic semigroup in $E$ (see e.g. [25, $\S 1.18]$ ). The the equation (5.3) have a solution $\hat{u}=U_{\eta, \mu}(y) \hat{\psi}(\eta)$ on $(0, \infty)$, where

$$
U_{\eta, \mu}(y)=\exp \left\{-\lambda(\eta, \mu) A_{\mu}^{\frac{1}{2}}(\eta) y\right\} \hat{\psi}(\eta)
$$

and $\lambda(\eta, \mu)$ is a root of (5.7) with positive real part. Then from the above expresion and the properties of analytic semigroups we get the uniform estimate

$$
\begin{gathered}
\|A \hat{u}\|_{h^{\alpha}\left(\mathbb{R}_{+} ; E\right)}+\sum_{j=0}^{2}|\mu|^{2-j}\left\|\hat{u}^{(j)}\right\|_{h^{\alpha}\left(\mathbb{R}_{+} ; E\right)} \leq \\
C_{1} \sup _{\eta \in[0,1]}\left\|\eta^{1-\alpha} A U(\eta) \hat{\psi}(., \eta)\right\|_{h^{\alpha}\left(\mathbb{R}_{+} ; E\right)} \leq C\|\hat{\psi}\|_{h^{2, \alpha}(A)},
\end{gathered}
$$

where $U(y)$ is a semigroup generated by $-A$.

Lemma 5.2. Assume the Condition 3.1 is satisfied. Then operator functions $q_{0}(\eta, y)$ and $q_{j}(\eta, y)$ are Fourier multipliears in $h^{\alpha}(\mathbb{R} ; E)$ uniformly with respect to $y \in \mathbb{R}_{+}$.

Proof. In view of (5.6) and the Remark 5.1 we get that

$$
\begin{aligned}
\sup _{\eta \in \mathbb{R}, y \in \mathbb{R}_{+}}(1+|\eta|)^{\frac{1}{2}}\left\|\frac{d}{d \eta}\left[q_{0}(\eta, y)\right]\right\|_{B(E)} \leq C_{1}, \\
\sup _{\eta \in \mathbb{R}, y \in \mathbb{R}_{+}}(1+|\eta|)^{\frac{1}{2}}\left\|\frac{d}{d \eta}\left[q_{j}(\eta, y)\right]\right\|_{B(E)} \leq C_{2},
\end{aligned}
$$

Then by using the multiplier results in [5] we can prove that the operator function $q_{0}(., y), q_{j}(., y)$ are multipliers in $h^{\alpha}(\mathbb{R} ; E)$ uniformly in $y \in \mathbb{R}_{+}$and $\mu \in \mathbb{R}$. 
Let

$$
\Phi_{0}(y)=\left\|q_{0}(., y)\right\|_{M\left(h^{\alpha}(\mathbb{R} ; E)\right)}, \Phi_{j}(y)=\left\|q_{j}(., y)\right\|_{M\left(h^{\alpha}(\mathbb{R} ; E)\right)} .
$$

Lemma 5.3. Assume the Condition 3.1 is satisfied. Then

$$
\Phi_{0}(y) \rightarrow 0, \Phi_{j}(y) \rightarrow 0 \text { as } y \rightarrow \infty .
$$

Proof. Indeed, by properties of Fourier multiplier operators from in $h^{\alpha}(\mathbb{R} ; E)$ and the theory of analytic semigroups there exists $\omega>0$ such that we have

$$
\begin{array}{r}
\left\|q_{0}(., y)\right\|_{M\left(h^{\alpha}(\mathbb{R} ; E)\right)} \leq C_{1}(|\lambda(\eta, \mu)| y)^{-1} \exp \{-\omega|\lambda(\eta, \mu)| y\}, \\
\left\|q_{j}(., y)\right\|_{M\left(h^{\alpha}(\mathbb{R} ; E)\right)} \leq C_{2}|\mu|^{2-j}\left(1+\eta^{2}\right)^{\frac{1}{2}} \exp \{-\omega|\lambda(\eta, \mu)| y\} .
\end{array}
$$

By estimates (5.12) and Remark 5.1 we obtain the assertion.

Proof of Theorem 5.1. By Lemma 5.1 the problem (5.1) - (5.2) has a solution

$$
u\left(x_{1}, x_{2}\right)=\left(\tilde{K}_{0}+\mu^{2}\right) \psi=F^{-1} N_{\mu}\left(\eta, x_{2}\right) \hat{\psi}(\eta) .
$$

By Lemmas 5.2, 5.3 the operator-functions $q_{0}(\eta, y)$ and $q_{j}(\eta, y)$ are Fourier multipliears in $h^{\alpha}(\mathbb{R} ; E)$ uniformly with respect to $y \in \mathbb{R}_{+}$. Then from the estimate 5.11 we obtain trhe assertion.

Here $K_{0 \mu}=K_{0 \mu}\left(x_{0}\right)$ denotes the inverse of operator $\tilde{K}_{0}\left(x_{0}\right)+\mu^{2}$, i.e.

$$
K_{0 \mu}\left(x_{0}\right)=\left[\tilde{K}_{0}\left(x_{0}\right)+\mu^{2}\right]^{-1} .
$$

Result 5.1. From Theorem 5.1 we obtain that the opertor $u \rightarrow K_{0 \mu} u$ is bounded from $h^{2, \alpha}(A)$ into $\tilde{Y}$ and the following estimate holds

$$
\begin{gathered}
\sum_{j=0}^{2}|\mu|^{2-j}\left\|\frac{\partial^{j} K_{0 \mu}}{\partial x_{1}^{j}}\right\|_{B\left(h^{2, \alpha}(A), \tilde{X}\right)}+\sum_{i, j=1}^{2}\left\|\frac{\partial^{2} K_{0 \mu}}{\partial x_{i} \partial x_{j}}\right\|_{B\left(h^{2, \alpha}(A), \tilde{X}\right)}+ \\
\left\|A K_{0 \mu}\right\|_{B\left(h^{2, \alpha}(A), \tilde{X}\right)} \leq C .
\end{gathered}
$$

Consider now the BVP

$$
\begin{aligned}
B\left(x_{0}\right) u & =\sum_{i, j=1}^{2}-a_{i j} \frac{\partial^{2} u}{\partial x_{i} \partial x_{j}}+A u+\mu^{2} u=0, x \in R_{+}^{2}, \\
B_{0}\left(x_{0}\right) u & =\left.\left[b_{1} \frac{\partial}{\partial x_{1}} u(x)+b_{2} \frac{\partial}{\partial x_{2}} u(x)\right]\right|_{x_{2=0}}=\psi\left(x_{1}\right),
\end{aligned}
$$

where

$$
A=A(g)\left(x_{0}, 0\right), a_{i j}=a_{i j}(g)\left(x_{0}, 0\right), b_{i}=b_{i j}(g)\left(x_{0}, 0\right)
$$

and $a_{i j}(g)$ are defined by (2.7).

Here,

$$
a_{1}(\eta, \mu)=i b_{1}\left(x_{0}\right) \eta-b_{2}\left(x_{0}\right) \lambda(\eta, \mu) .
$$




\section{Regularity properties of abstract elliptic operator with constant coefficients}

Consider the operator

$$
u \rightarrow O_{1} u=u \rightarrow O_{1}(g) u=B_{0}(g) K(g) u
$$

Here, $O_{10}$ denotes the constant coefficients version of $O_{1}$ fixed in $\left(x_{0}, 0\right)$ by (5.13), i.e.

$$
u \rightarrow O_{10} u=O_{1}\left(x_{0}, 0\right)=B_{0} K(g)\left(x_{0}, 0\right) u .
$$

Here,

$$
\begin{gathered}
O_{1 \mu} u=\left(O_{1}+\mu^{2}\right) u, O_{10 \mu} u=\left(O_{10}+\mu^{2}\right) u, \\
K_{0}=K\left(g\left(x_{0}, 0\right)\right), K_{\mu}(g)=\left[\tilde{K}(g)+\mu^{2}\right]^{-1} .
\end{gathered}
$$

We show the following result:

Theorem 6.1. Assume the Condition 3.1 is satisfied. Then the operator $u \rightarrow\left(O_{10}+\mu^{2}\right) u$ is an isomorphisim from $h^{2, \alpha}(A)$ onto $h^{1, \alpha}(A)$. Moreover, the following uniform estimates hold

$$
\begin{gathered}
\sum_{i=0}^{1}|\mu|^{2-i}\left\|\frac{\partial^{i} u}{\partial x_{1}^{i}}\right\|_{h^{1, \alpha}(A)}+\|A u\|_{h^{1, \alpha}(A)} \leq C\left\|\left(O_{10}+\mu^{2}\right) u\right\|_{h^{2, \alpha}(A)}, \\
C_{1}\|u\|_{h^{2, \alpha}(A)} \leq\left\|O_{20}+\mu_{0}^{2} u\right\|_{h^{1, \alpha}(A)} \leq C_{2}\|u\|_{h^{2, \alpha}(A)}
\end{gathered}
$$

for all $u \in h^{2, \alpha}(A)$ and $\mu>0$ with sufficiently large $\mu_{0}$.

Proof. In view of Lemma 3.1 and Theorem 5.1 it is clear to see that the solution of the problem

$$
\left(O_{10}+\mu^{2}\right) u=\psi
$$

has a unique solution $u$ exspressed as

$$
u=F^{-1} a_{1}(\eta, \mu) N_{\mu}\left(\eta, x_{2}\right) F \psi(\eta) .
$$

By lemmas 5.1-5.3 and by multiplier result in [5] we get that the operator functions $|\mu|^{2-i} \eta^{i} K\left(\eta, x_{2}\right)$ and $A K\left(\eta, x_{2}\right)$ are multipliers in $h^{\alpha}(\mathbb{R} ; E)$ uniformly with respect to $x_{2} \in \mathbb{R}_{+}$, where

$$
K\left(\eta, x_{2}\right)=a_{1}(\eta, \mu) N_{\mu}\left(\eta, x_{2}\right) .
$$

Hence, we obtain the estimate

$$
\sum_{i=0}^{1}|\mu|^{2-i}\left\|\frac{\partial^{i} u}{\partial x_{1}^{i}}\right\|_{h^{1, \alpha}(A)}+\left\|A_{0} u\right\|_{h^{1, \alpha}(A)} \leq C\left\|\left(O_{10}+\mu^{2}\right) u\right\|_{h^{2, \alpha}(A)} .
$$

Moreover, by definitions of the space $h^{2, \alpha}(A)$ we get (6.1) and the estimate

$$
\left\|O_{10}+\mu_{0}^{2} u\right\|_{h^{1, \alpha}(A)} \leq C_{2}\|u\|_{h^{2, \alpha}(A)} .
$$


Then in view of the above estimate, by reasoning as in the Theorem 5.1 we get the assertion and corresponding esimates.

By reasoning as in Theorem 6.1 we obtain

Theorem 6.2. Assume the Condition 3.1 is satisfied. Then the operator $O_{10}$ is positive and $-O_{10}$ is a generator of an analytic semigroup in $h^{1, \alpha}(A)$.

Proof. Indeed, for positivity of the operator $O_{10}$ in $h^{1, \alpha}(A)$ we need to show the estimate

$$
\left\|\left(O_{10}+\mu^{2}\right)^{-1}\right\|_{B\left(h^{1, \alpha}(A)\right)} \leq C \mu^{-2}
$$

i.e. we have to prove the estimate

$$
\|u\|_{h^{2, \alpha}(A)} \leq C \mu\left\|\left(O_{10}+\mu^{2}\right)^{-1} u\right\|_{h^{1, \alpha}(A)}
$$

for $u \in h^{2, \alpha}(A)$. By reasoning as the above we get that the function $\mu^{2} \eta a_{2}(\eta, \mu)$ is a multiplier in $h^{\alpha}(\mathbb{R} ; E)$ uniformly with respect to $x_{2} \in \mathbb{R}_{+}$, where

$$
a_{2}\left(\eta, x_{2}\right)=a_{1}(\eta, \mu) N_{\mu}\left(\eta, x_{2}\right) .
$$

So, the operator $O_{10}$ is positive in $h^{1, \alpha}(A)$. Then $-O_{10}$ is a generator of an analytic semigroup in $h^{1, \alpha}(A)$.

Here

$$
Q_{2} u=O_{2}(g) u=\partial B_{0}(g)[u, K(g) g]
$$

for $g \in h_{+}^{2+\alpha}$ and $u \in h^{2, \alpha}(A)$.

Consider the operator $O_{20}$ that is a constant coefficients version of $\mathrm{O}_{2}$ with fixed in $\left(x_{0}, 0\right)$ by $(5.15)$, i.e.

$$
O_{20}=O_{20}(g)=\partial B_{0}(g)[., K(g) g]\left(x_{0}, 0\right)
$$

for $g \in h^{2, \alpha}(A)$.

We prove the following result:

Theorem 6.3. Assume the Conditions 3.1 and 3.2 are satisfied. Then the operator $u \rightarrow\left(O_{20}+\mu^{2}\right) u$ is an isomorphisim from $h^{2, \alpha}(A)$ onto $h^{1, \alpha}(A)$. Moreover, the following estimate holds

$$
\begin{gathered}
\sum_{i=0}^{1}|\mu|^{2-i}\left\|\frac{\partial^{i} u}{\partial x_{1}^{i}}\right\|_{h^{1, \alpha}(A)}+\left\|A_{0} u\right\|_{h^{1, \alpha}(A)} \leq C\left\|\left(O_{20}+\mu^{2}\right) u\right\|_{h^{2, \alpha}(A)}, \\
C_{1}\|u\|_{h^{2, \alpha}(A)} \leq\left\|O_{20}+\mu_{0}^{2} u\right\|_{h^{1, \alpha}(A)} \leq C_{2}\|u\|_{h^{2, \alpha}(A)}
\end{gathered}
$$

for all $u \in h^{2, \alpha}(A)$, for sufficiently large $\mu_{0}$ and $\mu>0$.

Proof. By Lemma 3.1 we have

$$
O_{20} u=-\frac{\partial v}{\partial x_{1}} u_{x_{1}}+\frac{\partial v}{\partial x_{2}}\left(\Lambda_{1} u+\Lambda_{2} u\right),
$$


where

$$
\begin{gathered}
v=v(g)=K(g) g, \Lambda_{1} u=\frac{\left(1+g_{x_{1}}^{2}\right) u}{\nu+g}, \Lambda_{2} u=-\frac{1}{\nu+g} 2 g_{x_{1}} u_{x_{1}}, \\
O_{20} u=-\frac{\partial v_{0}}{\partial x_{1}} u_{x_{1}}+\frac{\partial v_{0}}{\partial x_{2}}\left(\Lambda_{1} u+\Lambda_{2} u\right), \\
v_{0}=v_{0}(g)=K(g)\left(x_{0}, 0\right) g, \Lambda_{1} u=\frac{\left(1+g_{x_{1}}^{2}\right) u}{\nu+g}, \Lambda_{2} u=-\frac{1}{\nu+g} 2 g_{x_{1}} u_{x_{1}},
\end{gathered}
$$

By using lemmas 5.1-5.3 we get that the operator function

$$
\left(\eta^{2}+\mu^{2}+A_{0}\right)\left[\eta+\Lambda(u) a_{1}(\eta, \mu)\right] N_{0 \mu}\left(\eta, x_{2}\right)
$$

is a multiplier in $h^{\alpha}(\mathbb{R} ; E)$ uniformly with respect to $x_{2} \in \mathbb{R}_{+}$, where

$$
A_{0 \mu}=A_{0 \mu}(\eta)=A+\mu^{2}+\eta^{2}, N_{0 \mu}(\eta, y)=A_{0 \mu}^{-\frac{1}{2}}(\eta) \exp \left\{-\lambda(\eta, \mu) A_{0 \mu}^{\frac{1}{2}}(\eta) y\right\} .
$$

Then by reasoning as in the Theorem 6.1 we obtain the assertion.

From Theorem 6.3 we obtain

Result 6.1. Assume the conditions 3.1 and 3.2 are satisfied. Then the operator $O_{20}$ is positive and $-O_{20}$ is a generator of an analityc semigroup in $h^{1, \alpha}(A)$.

Consider first of all, the BVP

$$
\begin{gathered}
-\sum_{k, j=1}^{2} a_{k j} \frac{\partial^{2} u}{\partial x_{k} \partial x_{j}}+\left(A+\mu^{2}\right) u+\mu^{2} u=V(x), \\
u\left(x_{1}, 0\right)=0, x=\left(x_{1}, x_{2}\right) \in R_{+}^{2},
\end{gathered}
$$

where $A=A(g)\left(x_{0}, 0\right), a_{k j}=a_{k j}(g)\left(x_{0}, 0\right)$ and $a_{k j}(g)$ are defined by (5.15).

Let $S_{0}=S(g)\left(x_{0}, 0\right)$ denotes the realizasion operator in $\tilde{X}$ generated by (6.7) $-(6.8)$ for $\mu=0$, i.e.

$$
D\left(S_{0}\right)=\tilde{Y}, S_{0} u=-\sum_{k, j=1}^{2} a_{k j} \frac{\partial^{2} u}{\partial x_{k} \partial x_{j}}+A u .
$$

From [4, Theorem 2] we obtain the following:

Result 6.2. Assume the Condition 3.1 is satisfied. Then;

(1) problem $(6.7)-(6.8)$ for sufficientli large $\mu>0$ has a unique solution $u \in \tilde{Y}$ for $V \in \tilde{X}$;

(2) the uniform coercive estimate holds

$$
\sum_{i=0}^{1}|\mu|^{2-i}\left\|\frac{\partial^{i} u}{\partial x_{1}^{i}}\right\|_{\tilde{X}}+\sum_{k, j=1}^{2}\left\|\frac{\partial^{2} u}{\partial x_{k} \partial x_{j}}\right\|_{\tilde{X}}+\|A u\|_{X} \leq C\|V\|_{\tilde{X}} ;
$$


(3) the operator $S_{0}$ is a positive and $-S_{0}$ is a generator of an analytic semigrop in $h^{1, \alpha}(A)$.

The estimate (6.9) particularly, implies that $\left(S_{0}+\mu^{2}\right)^{-1} \in B(\tilde{X}, \tilde{Y})$.

Consider the inhomogenous problem

$$
\begin{gathered}
-\sum_{k, j=1}^{2} a_{i j} \frac{\partial^{2} u}{\partial x_{k} \partial x_{j}}+A u+\mu^{2} u=V(x), \\
\gamma u=u\left(x_{1}, 0\right)=\psi\left(x_{1}\right), x=\left(x_{1}, x_{2}\right) \in R_{+}^{2} .
\end{gathered}
$$

Theorem 6.4. Assume the conditions 3.1 and 3.2 are satisfied. Then the operator $u \rightarrow G_{0} u=\left\{L_{0} u, \gamma u\right\}$ is an isomorphisim from $\tilde{Y}$ onto $\tilde{X} \times h^{2, \alpha}(A)$.

Proof. From definition of $\tilde{X}, \tilde{Y}, h^{2, \alpha}(A)$, from expresion of $L_{0}$ and by virtue of trace result in $\tilde{Y}[15, \mathrm{Ch} .2]$ we get that

$$
\left\|G_{0} u\right\|_{\tilde{X} \times h^{1, \alpha}(A)}=\left\|L_{0} u\right\|_{\tilde{X}}+\|\gamma u\|_{h^{2, \alpha}(A)} \leq C\|u\|_{\tilde{Y}},
$$

i.e. the operator $G_{0}$ is bounded linear from $\tilde{Y}$ into $\tilde{X} \times h^{2, \alpha}(A)$. Hence, in view of Banach theorem it is sufficient to show that the operator $G_{0}$ is inective and surjective from $\tilde{Y}$ onto $\tilde{X} \times h^{2, \alpha}(A)$. From Theorem 5.1 we obtain that the corresponding homogenous problem $L_{0} u=0, \gamma u=0$ has a zero solution, i.e. the operator $G_{0}$ is inective. So, it remain to show that this operator is surjective. By Theorem 5.1 we obtain that problem $L_{0} u=0, \gamma u=\psi$ has a solution $u_{1} \in$ $\tilde{Y}$ for all $\psi \in h^{2, \alpha}(A)$. Moreover, from the Result 6.2 we get that problem $L_{0} u=V, \gamma u=0$ has a solution $u_{2} \in \tilde{Y}$ for all $V \in \tilde{X}$. Then $u=u_{1}+u_{2}$ is a solution of $(6.10)-(6.11)$ that belongs to $\tilde{Y}$, i.e. the operator $G_{0}$ is surgective from $\tilde{Y}$ onto $\tilde{X} \times h^{2, \alpha}(A)$.

From Theorems 5.1 and 6.4 we obtain the following

Result 6.3. The soluion $u$ of the problem $(6.10)-(6.11)$ is exspressed as

$$
u(x)=S_{1, \mu} V+S_{2, \mu}\left(\psi-\gamma u_{1}\right),
$$

where

$$
S_{1, \mu} V=r_{+} F^{-1}\left(p(\xi)+A+\mu^{2}\right)^{-1} F \tilde{V}, S_{2, \mu} v=F^{-1} N_{\mu}\left(\xi_{1}, x_{2}\right) F v,
$$

here $r_{+}$is the restriction operator from $R^{2}$ into $R_{+}^{2}$ and $\tilde{V}=\tilde{V}\left(x_{1}, x_{2}\right)$ is an exstension of $V\left(x_{1}, x_{2}\right)$ on $R^{2}$, i.e

$$
\tilde{V}\left(x_{1}, x_{2}\right)=\left\{\begin{array}{c}
V\left(x_{1}, x_{2}\right), \text { if } x_{1}, x_{2} \in \bar{R}_{+}^{2} \\
V\left(x_{1},-x_{2}\right) \text { if } x_{1}, x_{2} \in R_{+}^{2}
\end{array}\right.
$$

$N_{\mu}\left(\xi_{1}, x_{2}\right)$-is operator function defined by (5.8) and $u_{1}$ is a solution of the equation

$$
-\sum_{k, j=1}^{2} a_{i j} \frac{\partial^{2} u}{\partial x_{k} \partial x_{j}}+A u+\mu^{2} u=\tilde{V}(x), x \in R^{2} .
$$


Let $O_{3}=O_{3}(g)$ be the operator in (4.6) defined by

$$
O_{3} u=B_{0}(g) S(g) \partial B(g)[u, K(g) g]
$$

for $g \in h^{2, \alpha}(A)$ and $u \in Y$.

In view of Lemma 3.1 and Lemma 3.2 we get

$$
O_{3} u=B_{0}(g) S(g) \partial B(g)[u, v], v=v_{g}(x)=(K(g) g)(x),
$$

where

$$
\begin{gathered}
\partial B(g)[u, v]=\frac{2 \beta}{\nu+g}\left\{\left[\left(\frac{g_{x_{1}} u}{\nu+g}-u_{x_{1}}\right)\right] v_{x_{1} x_{2}}+\right. \\
\frac{1}{(\nu+g)^{2}}\left[\left(\frac{1}{\beta}+\beta g_{x_{1}}^{2}\right) u-\frac{\beta}{\nu+g} g_{x_{1}} u_{x_{1}}\right] v_{x_{2} x_{2}}+\partial A(g)[u, v]- \\
\left.\left[\frac{g_{x_{1}}^{2} u}{(\nu+g)^{2}}-\frac{g_{x_{1} x_{1}} u+4 g_{x_{1}} u_{x_{1}}}{2(\nu+g)}+\frac{u_{x_{1} x_{1}}}{2}\right] v_{x_{2}}\right\} \\
O_{3}=O_{3}(g)=\sum_{i=1}^{4} O_{3 i}(g) u, O_{3 i}(g) u=B_{0}(g) S(g) G_{i}(g),
\end{gathered}
$$

where

$$
\begin{gathered}
G_{i}(g)=G_{i}(g)[u, v], G_{1}(g)=\frac{2 \beta}{\nu+g}\left[\left(\frac{g_{x_{1}} u}{\nu+g}-u_{x_{1}}\right)\right] v_{x_{1} x_{2}}, \\
G_{2}(g)=\left[\frac{u}{(\nu+g)^{2}}\left(\frac{1}{\beta}+\beta g_{x}^{2}\right)-\frac{\beta}{\nu+g} g_{x_{1}} u_{x_{1}}\right] v_{x_{2} x_{2}}, \\
G_{3}(g)=\frac{2 \beta}{\nu+g} \partial A(g)[u, v], \\
G_{4}(g)=-\left[\left(\frac{g_{x_{1}}^{2}}{(\nu+g)^{2}}-\frac{g_{x_{1} x_{1}}}{2(\nu+g)}\right) u-\frac{4 g_{x_{1}} u_{x_{1}}}{2(\nu+g)}+\frac{u_{x_{1} x_{1}}}{2}\right] v_{x_{2}} .
\end{gathered}
$$

Consider the operator $O_{30}$ that is the constant coefficients version of $O_{3}$ fixed in $\left(x_{0}, 0\right)$ which defined by $(5.16)$, i.e. from the above equality and from (6.13) we get $O_{30 k}=B_{0}(g) S(g) G_{k}(g)\left(x_{0}, 0\right)$,

$$
O_{30} u=O_{30}(g) u=O_{301} u+O_{302} u+O_{303} u+O_{304} u,
$$

where

$$
\begin{gathered}
O_{30 i}=O_{3 i}\left(g\left(x_{0}, 0\right)\right) u \\
w(x)=w_{g}(x)=\frac{\beta}{\nu+g} \frac{\partial}{\partial x_{2}} v_{g}\left(x_{1}, x_{2}\right),\left(x_{1}, x_{2}\right) \in \Omega, w_{0}=w_{g}\left(x^{0}, 0\right)
\end{gathered}
$$


For $u \in h^{2, \alpha}(A)$ define the operator by

$$
P_{1} u\left(x_{1}, x_{2}\right)=\left(P_{1}\left(g, x_{1}^{0}\right) u\right)\left(x_{1}, x_{2}\right)=w_{g}(x) u_{x_{1} x_{1}} e^{-x_{2}} .
$$

For later purposes we need the following technical lemmas:

Lemma 6.1. Assume the conditions 3.1 and 3.2 are satisfied. Then:

(a) $P_{1} \in B\left(h^{2, \alpha}(A), \tilde{X}\right)$;

(b) There exists a positive constant $C=C(g)$ such that

$$
\left\|P_{1} u-w u_{x_{1} x_{1}}\right\|_{h^{\alpha}\left(\tilde{U}_{r} ; E\right)} \leq C r\|u\|_{h^{2, \alpha}(A)}
$$

for all $u \in h^{2, \alpha}(A), r \in(0,1)$, where

$$
\tilde{U}_{r}=\mathbb{R}_{+}^{2} \cap U_{r}\left(x_{1}^{0}, 0\right),
$$

here, $U_{r}\left(x_{1}^{0}, 0\right)$ denotes the two dimensional ball with radius $r$ centered at $\left(x_{1}^{0}, 0\right)$.

Proof. Indeed, from the expression (6.14) in view of the Theorem 5.1 and by virtue of trace theorem in $Y$ we get (a); Then by using the integral mean value theorem and the trace theorem we obtain

$$
\left\|P_{1} u-w u_{x_{1} x_{1}}\right\|_{h^{\alpha}\left(\tilde{U}_{r} ; E\right)} \leq\left\|\left(w e^{-x_{2}}-w\right) u_{x_{1} x_{1}}\right\|_{h^{\alpha}\left(\tilde{U}_{r} ; E\right)} \leq C r\|u\|_{h^{2, \alpha}(A)} .
$$

Let

$$
\partial A=\partial A(x)=\partial A(g)(x), A_{0}=\partial A(g)\left(x_{10}, 0\right) .
$$

Lemma 6.2. Assume the conditions 3.1 and 3.2 are satisfied. Then the operator $u \rightarrow\left(O_{304}+\mu^{2}\right) u$ is an isomorphisim from $h^{2, \alpha}(A)$ onto $h^{1, \alpha}(A)$. Moreover, the following estimate holds

$$
\begin{gathered}
\sum_{i=0}^{1}|\mu|^{2-i}\left\|\frac{\partial^{i} u}{\partial x_{1}^{i}}\right\|_{h^{1, \alpha}(A)}+\left\|A_{0} u\right\|_{h^{1, \alpha}(A)} \leq C\left\|\left(O_{304}+\mu^{2}\right) u\right\|_{h^{2, \alpha}(A)}, \\
C_{1}\|u\|_{h^{2, \alpha}(A)} \leq\left\|O_{304}+\mu_{0}^{2} u\right\|_{h^{1, \alpha}(A)} \leq C_{2}\|u\|_{h^{2, \alpha}(A)}
\end{gathered}
$$

for all $u \in h^{2, \alpha}(A)$ and for sufficiently large $\mu_{0}, \mu>0$, .

Proof. From the expression (6.14) and Lemma 6.1 by reasoning as in $\left[9\right.$, lemma 5.3] we get that the operator $u \rightarrow\left(P_{1}+\mu^{2}\right) u$ is an isomorphism from $\tilde{Y}$ onto $\tilde{X}$. The Theorem 6.4 implies that the operator $u \rightarrow\left[S_{x_{0}}(g)+\mu^{2}\right] G_{40} u$ is an isomorphism from $\tilde{Y}$ onto $\tilde{Y}$ for sufficiently large $\mu>0$. Then in view of trace theorem in $\tilde{Y}$ we get that the operator $u \rightarrow\left[O_{304}+\mu^{2}\right] u$ is an isomorphism from $\tilde{Y}$ onto $h^{1, \alpha}(A)$. Moreover, in view of Result 6.3 by reasoning as in the Theorem 6.1 we obtain the estimates (6.16) for all $u \in h^{2, \alpha}(A)$ and for $\mu>0$ with sufficiently large $\mu_{0}$. 
Lemma 6.3. Assume the conditions 3.1 and 3.2 are satisfied. Then the operator $u \rightarrow\left(O_{303}+\mu^{2}\right) u$ is an isomorphisim from $h^{2, \alpha}(A)$ onto $h^{1, \alpha}(A)$. Moreover, the following estimate holds

$$
\begin{gathered}
\sum_{i=0}^{1}|\mu|^{2-i}\left\|\frac{\partial^{i} u}{\partial x_{1}^{i}}\right\|_{h^{1, \alpha}(A)}+\left\|A_{0} u\right\|_{h^{1, \alpha}(A)} \leq C\left\|\left(O_{303}+\mu^{2}\right) u\right\|_{h^{2, \alpha}(A)}, \\
C_{1}\|u\|_{h^{2, \alpha}(A)} \leq\left\|O_{303}+\mu_{0}^{2} u\right\|_{h^{1, \alpha}(A)} \leq C_{2}\|u\|_{h^{2, \alpha}(A)}
\end{gathered}
$$

for all $u \in h^{2, \alpha}(A)$ and for sufficiently large $\mu_{0}, \mu>0$.

Proof. From the expression (6.14) by properties of positive operators we get that the map $u \rightarrow\left(G_{30}+\mu^{2}\right) u$ is an isomorphism from $\tilde{Y}$ onto $\tilde{X}$. The Theorem 6.4 implies that the operator $u \rightarrow\left[S_{x_{0}}(g)+\mu^{2}\right] G_{30} u$ is an isomorphism from $\tilde{Y}$ onto $\tilde{Y}$ for sufficiently large $\mu>0$. Then in view of trace theorem in $\tilde{Y}$ we get that the operator $u \rightarrow\left[O_{303}+\mu^{2}\right] u$ is an isomorphism from $\tilde{Y}$ onto $h^{1, \alpha}(A)$. Moreover, in view of Result 6.3 by reasoning as in the Theorem 6.1 we obtain the estimates (6.17) for all $u \in h^{2, \alpha}(A)$ and for $\mu>0$ with sufficiently large $\mu_{0}$. obtain

In a similar way as Lemma 6.1 and by reasoning as in $[9$, lemma 5.4] we

Lemma 6.4. Assume the conditions 3.1 and 3.2 are satisfied. Then the operator $u \rightarrow\left(O_{30 k}+\mu^{2}\right) u$ is an isomorphisim from $h^{2, \alpha}(A)$ onto $h^{1, \alpha}(A)$. Moreover, the following estimate holds

$$
\begin{gathered}
\sum_{i=0}^{1}|\mu|^{2-i}\left\|\frac{\partial^{i} u}{\partial x_{1}^{i}}\right\|_{h^{1, \alpha}(A)}+\left\|A_{0} u\right\|_{h^{1, \alpha}(A)} \leq C\left\|\left(O_{30 k}+\mu^{2}\right) u\right\|_{h^{2, \alpha}(A)}, \\
C_{1}\|u\|_{h^{2, \alpha}(A)} \leq\left\|O_{30 k}+\mu_{0}^{2} u\right\|_{h^{1, \alpha}(A)} \leq C_{2}\|u\|_{h^{2, \alpha}(A)}, k=1,2,
\end{gathered}
$$

for all $u \in h^{2, \alpha}(A)$ and $\mu>0$.

Theorem 6.5. Assume the conditions 3.1 and 3.2 are satisfied. Then the operator $u \rightarrow\left(Q_{30}+\mu^{2}\right) u$ is an isomorphisim from $h^{2, \alpha}(A)$ onto $h^{1, \alpha}(A)$. Moreover, the following estimate holds

$$
\begin{gathered}
\sum_{i=0}^{1}|\mu|^{2-i}\left\|\frac{\partial^{i} u}{\partial x_{1}^{i}}\right\|_{h^{1, \alpha}(A)}+\left\|A_{0} u\right\|_{h^{1, \alpha}(A)} \leq C\left\|\left(O_{30}+\mu^{2}\right) u\right\|_{h^{2, \alpha}(A)}, \\
C_{1}\|u\|_{h^{2, \alpha}(A)} \leq\left\|O_{30}+\mu_{0}^{2} u\right\|_{h^{1, \alpha}(A)} \leq C_{2}\|u\|_{h^{2, \alpha}(A)}
\end{gathered}
$$

for all $u \in h^{2, \alpha}(A)$ and $\mu>0$.

Proof. From the expressions $(6.13)-(6.14)$ and from lemmas 6.2-6.4 we get that the operator $u \rightarrow K_{1 x_{0}}(g) u$ is an isomorphism from $\tilde{Y}$ onto $\tilde{X}$. The Theorem 6.4 implies that the operator $u \rightarrow\left[S_{x_{0}}(g)+\mu^{2}\right] K_{1 x_{0}}(g) u$ is an isomorphism from $\tilde{Y}$ onto $\tilde{Y}$ for sufficiently large $\mu>0$. Then in view of trace 
theorem in $\tilde{Y}$ we get that the operator $u \rightarrow\left[O_{30}+\mu^{2}\right] u$ is an isomorphism from $\tilde{Y}$ onto $h^{1, \alpha}(A)$ for sufficiently large $\mu_{0}$ and $\mu>0$.

From Theorem 6.5 we obtain

Result 6.4. Assume the Conditions 3.1 and 3.2 are satisfied. Then the operator $O_{30}$ is positive and $-O_{30}$ is a generator of an analytic semigroup in $h^{1, \alpha}(A)$.

From Theorems 6.1, 6.3 and 6.5 we obtain

Result 6.5. Assume the Conditions 3.1 and 3.2 are satisfied. Then

$$
O_{k 0}+\mu_{0} \in H\left(h^{2, \alpha}(A), h^{1, \alpha}(A)\right), k=1,2,3
$$

for sufficiently large $\mu_{0}>0$.

In view of theorems $6.3,6.5$ and by lemmas $2.3,3.1$ and Theorem 3.1 we obtain

Result 6.6. Assume the Conditions 3.1 and 3.2 are satisfied. The the following estimate holds

$$
\left\|O_{20}(g)+O_{30}(g)+\mu_{0}\right\|_{B\left(h^{2, \alpha}(A), h^{1, \alpha}(A)\right)} \leq C\|g\|_{h^{2, \alpha}(A)}
$$

for all

$$
g \in B_{0} \subset h^{2, \alpha}(A)
$$

Let

$$
O_{0}=O_{01}+O_{02}+O_{03} .
$$

Now, we will show that

$$
O_{0} \in H\left(h^{2, \alpha}(A), h^{1, \alpha}(A)\right) .
$$

Theorem 6.6. Assume the Conditions 3.1 and 3.2 are satisfied. Suppose

$$
\left\|w_{0}\right\|_{D_{A}(\alpha)} \leq \frac{\alpha(g)(x)}{a_{22}(g)(x, 0)}=\frac{\alpha_{0}}{a_{22}} .
$$

Then the operator $u \rightarrow\left(Q_{0}+\mu^{2}\right) u$ is an isomorphisim from $h^{2, \alpha}(A)$ onto $h^{1, \alpha}(A)$. Moreover, the following estimate holds

$$
\begin{gathered}
\sum_{i=0}^{1}|\mu|^{2-i}\left\|\frac{\partial^{i} u}{\partial x_{1}^{i}}\right\|_{h^{1, \alpha}(A)}+\left\|A_{0} u\right\|_{h^{1, \alpha}(A)} \leq C\left\|\left(O_{0}+\mu^{2}\right) u\right\|_{h^{2, \alpha}(A)}, \\
C_{1}\|u\|_{h^{2, \alpha}(A)} \leq\left\|O_{0}+\mu_{0}^{2} u\right\|_{h^{1, \alpha}(A)} \leq C_{2}\|u\|_{h^{2, \alpha}(A)}
\end{gathered}
$$

for all $u \in h^{2, \alpha}(A)$ and for sufficiently large $\mu_{0}, \mu>0$. Particularly, the operator $O_{0}$ is positive and $-O_{0}$ is a generator of an analytic semigroup in $h^{1, \alpha}(A)$.

Proof. Indeed, by using Theorems 6.1, 6.3, 6.5, the Results 6.3, 6.5, by reasoning as in lemma 5.4, Theorem 5.6, Corollary 5.7 in [2] and the perturbation results for space $H\left(h^{2, \alpha}(A), h^{1, \alpha}(A)\right)$ we obtain the assertion. 
Let

$$
\begin{gathered}
W_{t}=\left\{g \in h_{+}^{2, \alpha}, \inf _{x \in(-\infty, \infty)}\left[\frac{t}{\nu+g} \frac{\partial}{\partial x_{2}} v_{g}(x, 0)+k_{g}(x)>0\right]\right\}, \\
k_{g}(x)=\frac{\alpha(g)(x)}{a_{22}(g)(x, 0)}, t \in[0,1] .
\end{gathered}
$$

Remark 6.1. Suppose that $g \in W_{1}$. Then

$$
w_{\pi}=w_{g}(x, 0)<\frac{\alpha_{0}}{a_{22}} \text { for } x \in(-\infty, \infty) .
$$

From Theorem 5.1 we know that there is a constant $M>0$ such that

$$
\|K(g)\|_{B\left(h^{2, \beta}(\Gamma ; H), h^{2, \beta}(\Omega ; H)\right)} \leq M, \beta \in(0, \alpha)
$$

for all $g \in h_{+}^{2, \beta}$ satisfying $\|g\|_{h^{2, \beta}} \leq \chi$. Now define

$$
k=\frac{(\eta-\chi)^{3} \chi}{2 M\left[1+(\eta+\chi)^{2}+\chi^{2}\right](1+\chi)^{2}} .
$$

\section{Coercive estimates for the linearization}

It is clear that $O_{0}=O_{10}+O_{20}+O_{30}$ may be viewed as a principal part of the linearization of $\partial O(g)$ with coefficients fixed in $\left(x^{0}, 0\right)$. Our main goal in this section is to prove that the operator $O$ belongs to the class

$$
H\left(h^{2, \alpha}(A), h^{1, \alpha}(A)\right) .
$$

We use the estimates of local operators in the preceding section to derive coercive estimates for the linearization operator $\partial O(g)$. Let

$$
\partial O(g)=O_{1}(g)+O_{2}(g)+O_{3}(g),
$$

where

$$
\begin{gathered}
O_{1}(g)=B_{0}(g) K(g), O_{2}(g)=\partial B_{0}(g)[., K(g) g], \\
O_{3}(g)=-B_{0}(g) S(g) \partial B(g)[., K(g) g] .
\end{gathered}
$$

Given $g \in h_{+}^{2, \alpha}$ and $t \in[0,1]$, set

$$
\partial O^{t}(g)=O_{1}(g)+t \partial B_{0}(g)[., K(g) g]-t B_{0}(g) S(g) \partial B(g)[., K(g) g]
$$

and observe that

$$
\partial O^{1}(g)=\partial O(g) .
$$

Let $\delta>0$ be given and let $\left\{V_{j}, \varphi_{j}, j \in \mathbb{N}\right\}$ denote $\delta$-localizasion sequence for $S=\mathbb{R} \times\left(-\frac{\delta}{2}, \frac{\delta}{2}\right)$, the covering $\left\{V_{j}, j \in \mathbb{N}\right\}$ has finite multiplicity, diam $U_{j}<\delta$, 
and $\left\{V_{j}, \varphi_{j}, j \in \mathbb{N}\right\}$ is a partition of unity on $S$ with $\sum_{j \in \mathbb{N}} \varphi_{j}(x) \equiv 1$. Moreover, we fix $x_{1 j} \in \mathbb{R}$ such that $\left(x_{1 j}, 0\right) \in V_{j}, j \in \mathbb{N}$.

Here, we will prove the following result

Theorem 7.1. Assume the conditions 3.1 and 3.2 are satisfied. Suppose that $W_{0} \subset W_{1}$ is compact, $\beta \in(0, \alpha)$ and that $k>0$. Then there exist $\delta \in(0,1]$, a $\delta$-localization sequence $\left\{V_{j}, \varphi_{j}, j \in \mathbb{N}\right\}, \beta \in(0, \alpha)$ and a positive constant $C=C\left(W_{0}, M, \delta\right)$ such that

$$
\left\|\left[\varphi_{j} \partial O_{t}(g)-O_{\pi}\left(g, x_{1 j}\right) \varphi_{j}\right] v\right\|_{h^{1, \alpha}(A)} \leq k\left\|\varphi_{j} v\right\|_{h^{2, \alpha}(A)}+C\|v\|_{h^{2, \beta}(A)}
$$

for all $v \in h^{2, \alpha}(A), j \in \mathbb{N}, t \in[0,1]$ and $g \in W_{0}$.

For proving Theorem 7.1 we need some preparation. Let

$$
A_{j}=\partial A(g)\left(x_{1 j}, 0\right) .
$$

Consider the following equation

$$
(O(g)+\mu) u=f
$$

for

$$
f=[O(g)+\mu] u \in B_{1 p} .
$$

From (7.2) for $u_{j}=u \varphi_{j}, u \in D_{A}(\alpha)$ we get

$$
(O(g)+\mu) u_{j}=\sum_{k=1}^{3} O_{k}(g) u_{j}+\mu u_{j}=\sum_{k=1}^{3} F_{k j}+f \cdot \varphi_{j},
$$

where

$$
\begin{gathered}
F_{1 j}=\gamma\left[\left(b_{1} \frac{\partial \varphi_{j}}{\partial x_{1}}+b_{2} \frac{\partial \varphi_{j}}{\partial x_{2}}\right) U_{1}(g)\right], U_{1}(g)=K(g) u \\
F_{2 j}=\gamma\left[-\frac{\partial v}{\partial x_{1}} \frac{\partial \varphi_{j}}{\partial x_{1}}+\frac{2 g_{x_{1}}}{\nu+g} \frac{\partial v}{\partial x_{2}} \frac{\partial \varphi_{j}}{\partial x_{1}}\right] u, v=v(g)=K(g) g \\
F_{3 j}=\gamma\left[\left(b_{1} \frac{\partial \varphi_{j}}{\partial x_{1}}+b_{2} \frac{\partial \varphi_{j}}{\partial x_{2}}\right) U_{3}(g)\right], U_{3}(g)=S(g) \partial B(g)[u, K(g) g] .
\end{gathered}
$$

By freezing in (7.3) coefficients at points $\left(x_{1 j}, 0\right)$ we have localized equations

$$
\left(O\left(x_{1 j}\right)+\mu\right) u_{j}=F_{j},
$$

where

$$
\begin{gathered}
F_{j}=\sum_{k=1}^{3} F_{k j}+f \cdot \varphi_{j}+\sum_{k=1}^{3}\left(O_{k j}-O_{j}\right) u_{j} \\
\left(O_{1 j}-O_{1}\right) u_{j}=B_{0}(g)\left[K_{j}-K(g)\right] u_{j}+\left[B_{0 j}-B_{0}(g)\right] K_{j} u_{j} \\
\left(O_{2 j}-O_{2}\right) u_{j}=-\left(\frac{\partial v_{j}}{\partial x_{1}}-\frac{\partial v}{\partial x_{1}}\right) \frac{\partial}{\partial x_{1}} u_{j}+\left(\frac{\partial v_{j}}{\partial x_{2}}-\frac{\partial v}{\partial x_{2}}\right)\left(\Lambda_{1}+\Lambda_{2}\right) u_{j}
\end{gathered}
$$




$$
\left(O_{3 j}-O_{3}\right) u_{j}=\sum_{i=1}^{4}\left(O_{3 i j}-O_{3 i}\right) u_{j}
$$

here

$$
O_{3 i j}=B_{0}(g) S(g) G_{i}(g)\left(x_{1 j}, 0\right),
$$

and $O_{3 i j}, G_{i}$ are defined as in (6.14); moreover,

$$
\begin{gathered}
O\left(x_{1 j}\right)=O\left(x_{1 j}, 0\right)=\sum_{k=1}^{3} O_{k}(g)\left(x_{1 j}\right), O_{k}\left(x_{1 j}\right)=O_{k}(g)\left(x_{1 j}, 0\right), \\
B_{0 j}=B_{0}(g)\left(x_{1 j}, 0\right), K_{j}=K(g)\left(x_{1 j}, 0\right), v_{j}=v_{j}(g)=K(g)\left(x_{1 j}, 0\right) g, \\
S_{j}=S(g) \partial B(g)[., K(g) g]\left(x_{1 j}, 0\right),
\end{gathered}
$$

$O_{k}\left(x_{1 j}\right)$ are local operators fixed at points $\left(x_{1 j}, 0\right)$ defined by equalities $(6.3)$, $(6.5),(6.14),(6.15)$, respectively. From expressions of $F_{j}, K(g)\left(x_{1 j}, 0\right)$ by using $(6.3),(6.5),(6.14),(6.15)$ we get that $F_{j} \in h^{1, \alpha}(A)$.

For proving the Theorem 7.1 we need the following lemmas:

Lemma 7.1. The operator $u \rightarrow\left(O\left(x_{1 j}\right)+\mu^{2}\right) u$ is an isomorphisim from $h^{2, \alpha}(A)$ onto $h^{1, \alpha}(A)$. Moreover, the following estimate holds

$$
\sum_{i=0}^{1}|\mu|^{2-i}\left\|\frac{\partial^{i} u}{\partial x_{1}^{i}}\right\|_{h^{1, \alpha}(A)}+\left\|A_{j} u\right\|_{h^{1, \alpha}(A)} \leq C\left\|\left(O\left(x_{1 j}\right)+\mu^{2}\right) u\right\|_{h^{1, \alpha}(A)}
$$

for all $u \in h^{2, \alpha}(A)$ and for sufficiently large $\mu>0$.

Proof. Consider the equation

$$
\left(O\left(x_{1 j}\right)+\mu^{2}\right) u=f .
$$

Then, by virtue of Theorem 6.6 we obtain that the operator $u \rightarrow\left(O\left(x_{1 j}\right)+\mu^{2}\right) u$ is an isomorphisim from $h^{2, \alpha}(A)$ onto $h^{1, \alpha}(A)$ and the estimate (7.7) holds.

Lemma 7.2. There is a positive $\varepsilon \in(0,1)$ such that the following local estimate holds

$$
\left\|\left(O_{1 j}-O_{1}\right) u_{j}\right\|_{h^{1, \alpha}(A)} \leq \varepsilon\left\|K_{j} u_{j}\right\|_{h^{1, \alpha}(A)} .
$$

Proof. From (7.6) we get

$$
\left\|\left(O_{1 j}-O_{1}\right) u_{j}\right\|_{h^{1, \alpha}(A)} \leq\left\|B_{0}(g)\left[K_{j}-K(g)\right] u_{j}\right\|_{h^{1, \alpha}(A)}+\left\|\left[B_{0 j}-B_{0}(g)\right] K_{j} u_{j}\right\|_{h^{1, \alpha}(A)} .
$$

Then by taking into account of expressions $B_{0}(g), B_{0 j}, K(g), K_{j}$ and in view of smoothness of coefficients, choosing $\delta$ sufficiently small we have

$$
\left\|\left(O_{1 j}-O_{1}\right) u_{j}\right\|_{h^{1, \alpha}(A)} \leq\left\|B_{0}(g)\left[K_{j}-K(g)\right] u_{j}\right\|_{h^{1, \alpha}(A)}+
$$




$$
\begin{gathered}
\left\|\left(b_{1}-b_{1 j}\right) \frac{\partial}{\partial x_{1}} K_{j} u_{j}\right\|_{h^{1, \alpha}(A)}+\left\|\left(b_{2}-b_{2 j}\right) \frac{\partial}{\partial x_{2}} K_{j} u_{j}\right\|_{h^{1, \alpha}(A)} \leq \\
\varepsilon\left(\left\|\frac{\partial}{\partial x_{1}} K_{j} u_{j}\right\|_{h^{1, \alpha}(A)}+\left\|\frac{\partial}{\partial x_{2}} K_{j} u_{j}\right\|_{h^{1, \alpha}(A)}\right) \leq \varepsilon\left\|K_{j} u_{j}\right\|_{h^{2, \alpha}(A)} .
\end{gathered}
$$

Lemma 7.3. There is a positive $\varepsilon \in(0,1)$ such that the following local estimate holds

$$
\left\|\left(O_{2 j}-O_{2}\right) u_{j}\right\|_{h^{1, \alpha}(A)} \leq \varepsilon\left\|u_{j}\right\|_{h^{1, \alpha}(A)} .
$$

Proof. From the (7.6) we get

$$
\begin{gathered}
\left\|\left(O_{2 j}-O_{2}\right) u_{j}\right\|_{h^{1, \alpha}(A)} \leq\left\|\left(\frac{\partial v_{j}}{\partial x_{1}}-\frac{\partial v}{\partial x_{1}}\right) \frac{\partial}{\partial x_{1}} u_{j}\right\|_{h^{1, \alpha}(A)}+ \\
\left\|\left(\frac{\partial v_{j}}{\partial x_{2}}-\frac{\partial v}{\partial x_{2}}\right)\left(\Lambda_{1}+\Lambda_{2}\right) u_{j}\right\|_{h^{1, \alpha}(A)} .
\end{gathered}
$$

Then, by using the smoothness of coefficients and choosing $\delta$ sufficiently small, we get the estimate (7.9) .

Lemma 7.3. There is a positive $\varepsilon \in(0,1)$ such that the following local estimate holds

$$
\left\|\left(O_{3 j}-O_{3}\right) u_{j}\right\|_{h^{1, \alpha}(A)} \leq \varepsilon\left\|u_{j}\right\|_{h^{1, \alpha}(A)} .
$$

Proof. From expressions $(6.13)-(6.14)$ we get

$$
\left\|\left(O_{3 j}-O_{3}\right) u_{j}\right\|_{h^{1, \alpha}(A)} \leq \sum_{i=1}^{4}\left\|\left(O_{3 i j}-O_{3 i}\right) u_{j}\right\|_{h^{1, \alpha}(A)} .
$$

Moreover, from expressions $O_{31}$ and $G_{1}$ in (6.14) by boundedness of functions $g, \nu, \beta$ we have

$$
\begin{gathered}
\left\|\left(O_{31 j}-O_{31}\right) u_{j}\right\|_{h^{1, \alpha}(A)} \leq \\
C\left\|\left(B_{0}(g) S(g)\left(x_{1 j}, 0\right)-B_{0}(g) S(g)(x)\right)\left(u_{j}+\frac{\partial u_{j}}{\partial x_{1}}\right)\right\|_{h^{1, \alpha}(A)} \leq \\
C\left\{\left\|\left[B_{0}(g) S(g)\left(x_{1 j}, 0\right)-B_{0}(g) S(g)(x)\right] u_{j}\right\|_{h^{1, \alpha}(A)}+\right. \\
\left\|B_{0}(g) S(g)(x)\left[u_{j}(x)-u_{j}\left(x_{1 j}, 0\right)\right]\right\|_{h^{1, \alpha}(A)}+ \\
\left\|\left[B_{0}(g) S(g)\left(x_{1 j}, 0\right)-B_{0}(g) S(g)(x)\right] \frac{\partial u_{j}}{\partial x_{1}}\right\|_{h^{1, \alpha}(A)}+ \\
\left\|B_{0}(g) S(g)(x)\left[\frac{\partial u_{j}}{\partial x_{1}}-\frac{\partial}{\partial x_{1}} u_{j}\left(x_{1 j}, 0\right)\right]\right\|_{h^{1, \alpha}(A)} \cdot
\end{gathered}
$$


Then by boundedness of operator $B_{0}(g) S(g)$, smoothness of coefficients, choosing $\delta$ sufficiently we obtain from the above

$$
\left\|\left(O_{31 j}-O_{31}\right) u_{j}\right\|_{h^{1, \alpha}(A)} \leq \varepsilon\left\|u_{j}\right\|_{h^{2, \alpha}(A)} .
$$

In a similar way, from expressions $O_{32}$ and $G_{2}$ in (6.14) we have

$$
\left\|\left(O_{32 j}-O_{32}\right) u_{j}\right\|_{h^{1, \alpha}(A)} \leq \varepsilon\left\|u_{j}\right\|_{h^{2, \alpha}(A)} .
$$

In view of the condition on the operator $\partial A(g)$, boundedness of operator $B_{0}(g) S(g)$, smoothness of coefficients, choosing $\delta$ sufficiently we obtain

$$
\left\|\left(O_{33 j}-O_{33}\right) u_{j}\right\|_{D_{A}(\alpha)} \leq \varepsilon\left\|u_{j}\right\|_{D_{A}(\alpha)} .
$$

Finally, from expressions $O_{34}$ an $G_{4}$ in (6.14) by boundedness of functions $g, \nu, \beta$ we have

$$
\begin{gathered}
\left\|\left(O_{34 j}-O_{34}\right) u_{j}\right\|_{h^{1, \alpha}(A)} \leq \\
C\left\|\left(B_{0}(g) S(g)\left(x_{1 j}, 0\right)-B_{0}(g) S(g)(x)\right)\left(u_{j}+\frac{\partial u_{j}}{\partial x_{1}}+\frac{\partial^{2} u_{j}}{\partial x_{1}^{2}}\right)\right\|_{h^{1, \alpha}(A)} \leq \\
C\left\{\left\|\left[B_{0}(g) S(g)\left(x_{1 j}, 0\right)-B_{0}(g) S(g)(x)\right] u_{j}\right\|_{h^{1, \alpha}(A)}+\right. \\
\left\|B_{0}(g) S(g)(x)\left[u_{j}(x)-u_{j}\left(x_{1 j}, 0\right)\right]\right\|_{h^{1, \alpha}(A)}+ \\
\left\|\left[B_{0}(g) S(g)\left(x_{1 j}, 0\right)-B_{0}(g) S(g)(x)\right] \frac{\partial u_{j}}{\partial x_{1}}\right\|_{h^{1, \alpha}(A)}+ \\
\left\|B_{0}(g) S(g)(x)\left[\frac{\partial u_{j}}{\partial x_{1}}-\frac{\partial}{\partial x_{1}} u_{j}\left(x_{1 j}, 0\right)\right]\right\|_{h^{1, \alpha}(A)}+ \\
\left\|\left[B_{0}(g) S(g)\left(x_{10}, 0\right)-B_{0}(g) S(g)(x)\right] \frac{\partial^{2} u_{j}}{\partial x_{1}^{2}}\right\|_{D_{A}(\alpha)}+ \\
\left\|B_{0}(g) S(g)(x)\left[\frac{\partial^{2} u_{j}}{\partial x_{1}^{2}}-\frac{\partial^{2}}{\partial x_{1}^{2}} u_{j}\left(x_{1 j}, 0\right)\right]\right\|\left\|_{B_{1 p}} \leq \varepsilon\right\|_{j} \|_{h^{1, \alpha}(A)} .
\end{gathered}
$$

Then the estiamate (7.10) is obtained from (7.12) - (7.15).

Now, we can prove the Theorem 7.1.

Proof of Theorem 7.1. By virtue of Theorem 6.6 the following estimate holds

$$
\sum_{i=0}^{1}|\mu|^{2-i}\left\|\frac{\partial^{i} u_{j}}{\partial x_{1}^{i}}\right\|_{h^{1, \alpha}(A)}+\left\|A_{0} u_{j}\right\|_{h^{1, \alpha}(A)} \leq C\left\|F_{j}\right\|_{h^{1, \alpha}(A)}
$$

for all solution $u_{j} \in h^{2, \alpha}(A)$ of the equation (7.5). 
Whence, using smoothness of coefficients of equations (7.3) - (7.6), in view of Lemmas 7.1-7.3 for $\mu$ with sufficiently large $\operatorname{Re} \mu>0$ we get

$$
\left\|F_{j}\right\|_{h^{1, \alpha}(A)} \leq \varepsilon\left[\sum_{i=0}^{1}|\mu|^{2-i}\left\|\frac{\partial^{i} u_{j}}{\partial x_{1}^{i}}\right\|_{h^{1, \alpha}(A)}+\left\|A_{j} u_{j}\right\|_{h^{1, \alpha}(A)}\right]+\left\|f \cdot \varphi_{j}\right\|_{h^{1, \alpha}(A)}
$$

Moreover, by appling the microlocal analysis reasoning as in theorems 1, 2 in [21] and in theorem 6.2 and as in [9, Lemma 6. 5-6.7] we obtain the same estimates for corresponding commutators operators. Then from this and from (7.16), (7.17) we get the assertion.

From the Theorem 7.1 by microlocal analysis reasoning as in theorem 6.2 and corollary 6.3 in [9] we obtain

Corollary 7.1. Assume the conditions $3.1,3.2$ are satisfied and $K \subset W$ is compact. Then there exist positive constants $\mu_{0}$ and $C=C(K)$ such that

$$
\|v\|_{h^{2, \alpha}(A)}+|\mu|\|v\|_{h^{1, \alpha}(A)} \leq C\left\|\left(\mu+\partial O_{t}(g)\right) v\right\|_{h^{1, \alpha}(A)}
$$

for all $v \in h^{2, \alpha}(A), g \in K, t \in[0,1]$ and $\mu \in\left\{z: \operatorname{Re} z>\mu_{0}\right\}$.

Corollary 7.2. Let $g \in W$ and $t \in[0,1]$ be given. Then

$$
\partial O_{t}(g) \in H\left(h^{2, \alpha}, h^{1, \alpha}\right) .
$$

Now, by using Theorem 7.1 we can prove Theorem 1 .

Proof of Theorem 1: Let $f_{0} \in V_{\nu}$ be given and set $g_{0}=f_{0}-\nu$. Observe that $g_{0} \in W_{\alpha, 1}=W$. It follows from Lemma 4.1 that we only have to prove that there exist $t_{+}>0$ and a unique maximal classical solution of $(1.1)-(1.2)$ on $\left[0, t_{+}\right)$satisfying

$$
\lim _{t \rightarrow t^{+}}\|g(t, .)\|_{h^{2, \alpha}}=\infty, \lim _{t \rightarrow t^{+}} \inf _{v \in \partial W}\|f(t, .)-v\|_{h^{2, \alpha}}=0
$$

if $t_{+}<\infty$ and $g \in C_{b}\left(\left[0, t_{+}\right) ; W\right)$.

By reasoning as [9, Lemma 5.10] It follows that $W$ is an open subset of $h_{+}^{2, \alpha}$. Hence, thanks to Lemma 4.2 and Corollary 7.2, we know that $O \in H\left(W, h^{1, \alpha}\right)$ and that

$$
\partial O(g) \in H\left(h^{2, \alpha}, h^{1, \alpha}\right), g \in W .
$$

Let now $\beta \in(0, \alpha)$ be fixed and observe that $W \subset W_{\beta, 1}$. Thus the very same arguments as above also ensure that

$$
\partial O(g) \in H\left(h^{2, \beta}, h^{1, \beta}\right), g \in W .
$$

It is not difficult to see that the maximal $h^{1, \alpha}$-realization of $\partial O(g) \in B\left(h^{2, \beta}, h^{1, \beta}\right)$ for $g \in W$, is just the linear operator in (7.19). Note that

$$
\left(h^{1, \beta}, h^{2, \beta}\right)_{\alpha-\beta, \infty}=h^{1, \alpha}
$$


where $(., .)_{\alpha-\beta, \infty}$ denotes the real interpolation. Consequently, invoking Theorem 2.3 in [24], we find that

$$
\partial O(g) \in M_{1}\left(h^{2, \alpha}, h^{1, \alpha}\right), g \in W,
$$

where $M\left(E_{1}, E_{2}\right)$ denotes the class of all operators in s having the property of maximal regularity in the sense of Da Prato and Grisvard [6]. The existence of a unique maximal classical solution of $(\mathrm{E}) \mathrm{g}$ o and the property of a smooth semiflow on $W$ can now be obtained along the lines of the proofs of Proposition 3.5 and Theorem 3.2 in [24].

Finally suppose that $t_{+}<\infty, g \in C_{b}\left(\left[0, t_{+}\right) ; W\right)$ and that $(7.18)$ is not true. Then $g_{1}=\lim _{t \rightarrow t_{+}} g(t)$ exists in $W$. Hence taking $\quad g_{1}$ as initial value in (4.2) one easily constructs a solution $\bar{g}$ of (4.2) for initial date $g_{1}$ extending $g$. This contradicts the maximality of $g$.

\section{References}

1. Amann H., Maximal regularity for non-autonomous evolution equations. Advanced Nonlinear Studies, (2004) 4, 417-430.

2. Agarwal R., Bohner M., Shakhmurov V. B., Linear and nonlinear nonlocal boundary value problems for differential operator equations, Appl. Anal., (2006), 85(6-7), 701-716.

3. Ashyralyev A, Cuevas. C and Piskarev S., On well-posedness of difference schemes for abstract elliptic problems in spaces, Numer. Func. Anal. Opt., (2008)29, (1-2), 43-65.

4. Anosov V. P., Sobolevskii P. E., Coercive solvability boundary value problems for elliptic equations of the second orders in Banach spaces, I, II, Differ. Uravn., (7)11, 2030-2044, (7)12 (1971), 2191-2198.

5. Arendt W., Batty C., Bu S., Fourier multipliers for Holder continuous functions and maximal regularity, Studia Mathematica 160 (1) (2004), $1-29$.

6. Da Prato P., Grisvard, P., Equations d'évolution abstraites nonlinairés de type parabolique, Ann. Mat. Pura Appl., (4) 120, 329-396 (1979).

7. Dore, G., $L_{p}$-regularity for abstract differential equations. In: Functional Analysis and Related Topics, H. Komatsu (ed.), Lecture Notes in Math. 1540. Springer, 1993.

8. Denk R., Hieber M., Prüss J., R-boundedness, Fourier multipliers and problems of elliptic and parabolic type, Mem. Amer. Math. Soc. (2003), 166 (788), 1-111. 
9. Escher J., Simonett G., Maximal regularity for a free boundary problem, NoDEA 2 (1995) 463-510.

10. Favini A., Shakhmurov V., Yakubov Y., Regular boundary value problems for complete second order elliptic differential-operator equations in UMD Banach spaces, Semigroup Form, (2009), 79 (1), 22-54.

11. Favini, A., Yagi, A., Degenerate Differential Equations in Banach Spaces, Taylor \& Francis, Dekker, New-York, 1999.

12. Goldstain J. A., Semigroups of Linear Operators and Applications, Oxford University Press, Oxfard, 1985.

13. M. Girardi and L. Weis, Operator-valued Fourier multiplier theorems on Besov spaces, Mathematische Nachrichten, Math. Nachr. 251 (2003), $34-51$.

14. Krein S. G., Linear Differential Equations in Banach space, American Mathematical Society, Providence, 1971.

15. Lunardi A., Analytic Semigroups and Optimal Regularity in Parabolic Problems, Birkhauser, 2003.

16. Lions J. L., Peetre J., Sur one classe d'espases d'interpolation, IHES Publ. Math. (1964)19, 5-68.

17. Shklyar, A.Ya., Complete second order linear differential equations in Hilbert spaces, Birkhauser Verlak, Basel, 1997.

18. Sobolevskii P. E., Coerciveness inequalities for abstract parabolic equations, Dokl. Akad. Nauk, (1964), 57(1), 27-40.

19. Shahmurov R., On strong solutions of a Robin problem modeling heat conduction in materials with corroded boundary, Nonlinear Anal. Real World Appl., (2011),13(1), 441-451.

20. Shahmurov R., Solution of the Dirichlet and Neumann problems for a modified Helmholtz equation in Besov spaces on an annuals, J. Differential Equations, 2010, 249(3), 526-550.

21. Shakhmurov V. B., Maximal regular abstract elliptic equations and applications, Siberian Mathematical Journal, V.51, no 5, 935-948, 2010.

22. Shakhmurov V. B., Degenerate differential operators with parameters, Abstr. Appl. Anal., (2007), 2006, 1-27.

23. Shakhmurov V. B., Separable anisotropic differential operators and applications, J. Math. Anal. Appl., 327(2) 2006, 1182-1201.

24. Simonett, G. Quasilinear parabolic equations and semiflows. In Evolution Equations, Control Theory, and Biomathematics, Lecture Notes in Pure and Appl. Math. M. Dekker, New York, 523-536, (1994). 
25. Triebel H., Interpolation Theory, Function Spaces, Differential Operators, North-Holland, Amsterdam, 1978.

26. Triebel H., Theory of Function Spaces II, Birkhuser-Verlag, Basel, 1992.

27. Weis L, Operator-valued Fourier multiplier theorems and maximal $L_{p}$ regularity, Math. Ann., (2001), 319, 735-758.

28. Yakubov S. and Yakubov Ya., Differential-Operator Equations. Ordinary and Partial Differential Equations, Chapman and Hall /CRC, Boca Raton, 2000 . 